\title{
Dynamical Instability Determines the Effect of Ongoing Noise on Neural Firing
}

\author{
David E. O’Gorman, ${ }^{1,2,3}$ John A. White, ${ }^{3}$ and Christopher A. Shera ${ }^{1,2,4}$ \\ ${ }^{1}$ Eaton-Peabody Laboratory of Auditory Physiology, Massachusetts Eye and Ear Infirmary, 243 Charles Street, Boston, MA \\ 02114, USA \\ ${ }^{2}$ Speech and Hearing Bioscience and Technology Program, Harvard-MIT Division of Health Sciences and Technology, \\ Cambridge, MA 02139, USA \\ ${ }^{3}$ Department of Biomedical Engineering, Center for BioDynamics, and Neuronal Dynamics Laboratory, Boston University, \\ Boston, MA 02215, USA \\ ${ }^{4}$ Department of Otology and Laryngology, Harvard Medical School, Boston, MA 02115, USA
}

Received: 16 March 2008; Accepted: 12 November 2008; Online publication: 24 March 2009

\begin{abstract}
At low stimulation rates, electrically stimulated auditory nerve fibers typically fire regularly, in lock-step to the applied stimulus. At high stimulation rates, however, these same fibers fire irregularly. Firing irregularity has been attributed to the random opening and closing of voltage-gated sodium channels at the spike generation site. We demonstrate, however, that the nonlinear dynamics of neural excitation and refractoriness embodied in the FitzHughNagumo (FN) model produce realistic firing irregularity at high stimulus rates, even in the complete absence of ongoing physiological noise. Indeed, we show that ongoing noise can actually regularize the response at low discharge rates. The degree of stimulus-dependent irregularity is determined not so much by the level of ongoing physiological noise as by the dynamical instability. Our work suggests that the dynamical instability, quantified by the Lyapunov exponent, controls neural sensitivity to input signals and to physiological noise, as well the amount of mutual desynchronization between similarly stimulated fibers. This instability, quantified by
\end{abstract}

Electronic supplementary material The online version of this article (doi:10.1007/s10162-008-0148-5) contains supplementary material, which is available to authorized users.

Correspondence to: David E. O'Gorman · Hearing Research Center, Department of Biomedical Engineering, Boston University, Boston, MA 02215, USA. Telephone: +857-2227443; fax: +617-3536766; email: ogorman@bu.edu the value of the Lyapunov exponent, may play a critical role in determining modulation sensitivity and dynamic range in cochlear implants.

Keywords: cochlear implant, electrical stimulation, chaos, firing irregularity, desynchronization, auditory nerve, Lyapunov exponent

The firing irregularity exhibited by electrically stimulated auditory nerve fibers is thought to be produced primarily by ongoing physiological noise (Dynes 1996; Morse and Evans 1996; Moss et al. 1996; Bruce et al. 1999; Rubinstein et al. 1999; Javel and Viemeister 2000; Litvak et al. 2001, 2003a, b, c; Matsuoka et al. 2001; Miller et al. 2001, 2008; Stocks et al. 2002; Hong and Rubinstein 2003; Zhang et al. 2007), in particular, by the thermally driven random opening and closing of voltage-gated ion channels in the nerve membrane (Sakmann and Neher 1983; White et al. 2000), especially the sodium channels (Lecar and Nossal 1971a, b; Sigworth 1980; Rubinstein 1995). However, noise alone is incapable of accounting for the dependence of the firing irregularity on the stimulation rate. For example, at low rates and stimulus amplitudes sufficiently above threshold, auditory nerve fibers fire regularly, in lock-step to the applied stimulus. At high rates, however, the same fibers fire 
irregularly (Moxon 1967; van den Honert and Stypulkowski 1987; Javel 1990; Javel and Viemeister 2000). Figure 1 shows an example of the irregular firing pattern produced by a high-rate $(5 \mathrm{kHz})$ periodic pulse train. Although ongoing physiological noise might account for such irregularity at high stimulation rates, the same explanation cannot account for its absence at low rates.

Besides ongoing noise, another mechanism for producing irregularity is dynamical instability or "chaos" (Eckmann and Ruelle 1985). Dynamical instability was first demonstrated computationally in a model of atmospheric convection (Lorenz 1963), and has since been shown to occur in neural models (Aihara et al. 1984; Ermentrout 1984; Feingold et al. 1988; Rajasekar and Lakshmanan 1988, 1994; Sato and Doi 1992; Rabinovitch et al. 1994; Doi and Sato 1995; Kaplan et al. 1996; Ichinose et al. 1998; Rinzel and Ermentrout 1998; Othmer and Xie 1999; Rabinovitch and Rogachevskii 1999; Coombes and Osbaldestin 2000; Guckenheimer and Oliva 2002; Kosmidis and Pakdaman 2006; Lin 2006) and in empirical results from the squid giant axon when it is stimulated electrically at high rates (Matsumoto et al. 1987; Takahashi et al. 1990; Kaplan et al. 1996). In this study, we use a mathematical model of neuralspike generation to investigate the relative importance of the level of ongoing noise and the degree of dynamical instability in producing the neural firing irregularity observed in electrically stimulated auditory nerve fibers. The degree of instability is quantified by the spike generator's Lyapunov exponent, a number that measures the rate of growth of the response to a small perturbation.

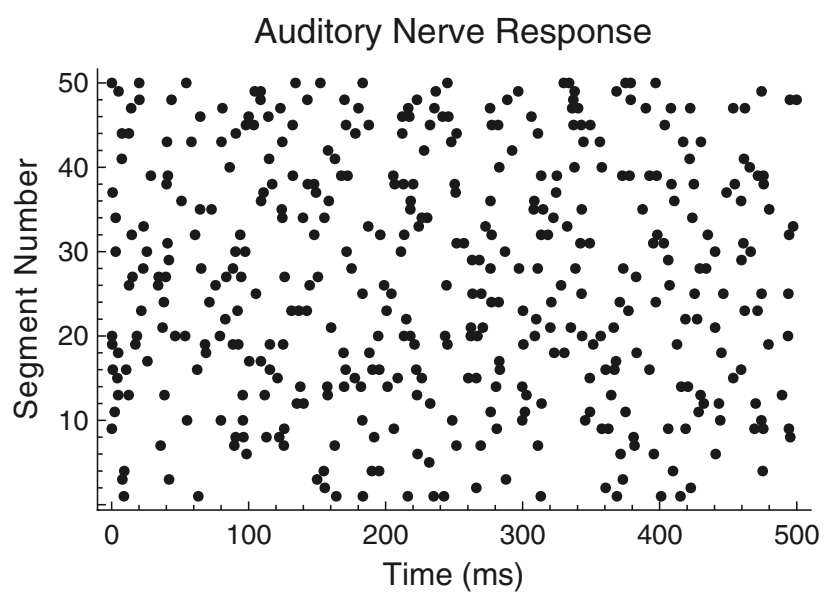

FIG. 1. The irregular response of a mammalian auditory nerve fiber to high-rate electrical stimulation. A dot raster plot showing the spike times of a mammalian auditory nerve fiber when stimulated by a $5-\mathrm{kHz}$ electrical pulse train. Dots indicate the time of occurrence of a spike within a 500-ms time window. Responses to 50 contiguous segments are stacked vertically in the figure. Auditory nerve data were collected by Litvak et al. (2003a).
The specific model we use is the FitzHughNagumo (FN) model, sometimes referred to as the Bonhoeffer-van der Pol model (Bonhoeffer 1948; FitzHugh 1961; Nagumo et al. 1963). This simple model approximates the time course of excitation and refractoriness predicted by more complex biophysical models such as those of the Hodgkin-Huxley class (Hodgkin and Huxley 1952; FitzHugh 1961; Kepler et al. 1992). The FN model has been used in many contexts, including applications involving electrical stimulation of the auditory nerve (Hochmair-Desoyer et al. 1984). Most significantly for this paper, previous studies have shown that the FN model captures the main features of the squid axon's response to highrate stimulation (Sato and Doi 1992; Rabinovitch et al. 1994; Doi and Sato 1995; Kaplan et al. 1996). For example, temporal spiking patterns predicted by the noise-free FN model (Fig. 3a of Sato and Doi 1992) are qualitatively similar to those recorded in squid axons (Fig. 6 of Takahashi et al. 1990). Indeed, although the FN model is a simplified approximation to the Hodgkin-Huxley ( $\mathrm{HH})$ model, it appears to capture the main features of the response of the squid axon to high-rate stimulation better than the $\mathrm{HH}$ model itself (Clay 1998; O'Gorman and Shera 2001).

\section{METHODS}

\section{The FitzHugh-Nagumo model}

The FitzHugh-Nagumo model consists of two coupled ordinary differential equations. The first determines the rapid time evolution of neural excitation $x(t)$ and the second determines the slower evolution of refractoriness $y(t)$ :

$$
\begin{gathered}
x^{\prime}(t)=c\left(x(t)-\frac{1}{3} x(t)^{3}-y(t)\right)+I(t), \\
y^{\prime}(t)=\frac{1}{c}(x(t)+a-b y(t)),
\end{gathered}
$$

where:

$$
1-2 b / 3<a<1, \quad 0<b<1, \quad b<c^{2}
$$

FitzHugh's analysis of the Hodgkin-Huxley equations has demonstrated that $x(t)$ approximates the combined dynamics of the membrane voltage and sodium-channel activation and that $y(t)$ approximates the combined dynamics of sodium-channel inactivation and potassium-channel activation (FitzHugh 1961, 1969). The conditions in Eq. (3) guarantee that there is a single resting condition and that the model is capable of producing action potentials.

The term $I(t)$ in Eq. (1) represents the current waveform used to excite the neuron. We use the 
simplest possible periodic pulse train, namely, a superposition of idealized Dirac delta functions $I(t)=\sum_{j=1}^{N_{\text {pulse }}} A \delta(t-j T)$, where $A$ is the stimulus amplitude,

$T$ is the interpulse time, and $N_{\text {pulse }}$ is the number of pulses in the pulse train (FitzHugh 1961). The use of delta-function pulses is justified by the briefness of the "biphasic" pulses used in the physiological studies of Litvak et al. (20-25 $\mu \mathrm{s} /$ phase) and Miller et al. (40 $\mu \mathrm{s} /$ phase) and by the fact that these delta-function pulses have very nearly the same effect as brief but finite duration pulses (FitzHugh 1961). The response of the FN model to biphasic pulses which consist of positiveand negative-going phases are considered in Section 3 of the Supplemental Computations.

The values of the system parameters used in this study are $a=0.753617, b=0.745338$, and $c=3.28076$. These values are the same as those used in a prior study that showed the FN model produces responses similar to that of the squid giant axon to rapid pulsatile stimulation (Kaplan et al. 1996).

To account for the faster kinetics of auditory nerve fibers compared to squid axons, time $t$ within the FN model description was scaled by a factor $\gamma \equiv$ $\hat{t}_{d} / t_{d} \simeq 0.205 \mathrm{~ms} / 3.66 \simeq 0.056 \mathrm{~ms}$, where $t_{d}$ is the action potential downstroke time in the original dimensionless units of FitzHugh (1961) and $\hat{t}_{d}$ is the action potential downstroke time of an auditory nerve fiber estimated from the neural recordings of Moxon (1967). For most of the simulations presented in this paper, the interpulse interval is $\hat{T} \equiv \gamma T=0.2 \mathrm{~ms}$, which closely approximates the interpulse time used in physiological experiments (Litvak et al. 2001, 2003a, b, c; Zhang et al. 2007; Miller et al. 2008) and in computational simulations (Rubinstein et al. 1999).

\section{The variational equations}

We quantify the degree of dynamical instability in the FN model by computing the Lyapunov exponent, a number that measures the rate of growth of the response to a small perturbation (Eckmann and Ruelle 1985). When the Lyapunov exponent is positive, the response to a perturbation grows with time and the system is dynamically unstable. The time evolution of the response to small perturbations is governed by the variational equations, which can be derived from Eqs. (1) and (2). The Lyapunov exponent is obtained by solving these variational equations.

To derive the variational equations, first consider a vector $\vec{X}_{0}=\left\{x_{0}, y_{0}\right\}$ with its tail at the origin of a twodimensional plane and its head at the coordinates $\left(x_{0}, y_{0}\right)$. This vector $\vec{X}_{0}$ represents the initial condition of the solution to the FN equations when driven by a high-rate pulse train. The solution of the driven FN
Eqs. (1) and (2), starting from this initial condition, is represented by the trajectory traced out by the head of the time-dependent vector $\vec{X}(t)=\{x(t), y(t)\}$, where, at time $t$ equal to zero, $\vec{X}(t)$ has the value $\vec{X}(0)=\vec{X}_{0}=\left\{x_{0}, y_{0}\right\}$. Such a trajectory $\vec{X}(t)$, starting at an initial condition of $\vec{X}_{0}=\{-0.52,-0.62\}$, is shown in Figure 2.

Now consider an infinitesimal perturbation $\overrightarrow{\mathcal{R}}=\left\{\mathcal{R}_{x}, \mathcal{R}_{y}\right\}$ applied to a point on the trajectory $\vec{X}(t)$. The perturbed condition (not shown) is then described by the vector $\vec{X}+\overrightarrow{\mathcal{R}}=\left\{x+\mathcal{R}_{x}, y+\mathcal{R}_{y}\right\}$. The variational equations describe the time evolution of the components of the perturbation vector $\overrightarrow{\mathcal{R}}=\left\{\mathcal{R}_{x}, \mathcal{R}_{y}\right\}$. If we define the right-hand side of Eqs. (1) and (2), respectively, as $F_{1}$ and $F_{2}$, then the equations governing the evolution of $\overrightarrow{\mathcal{R}}$ are

$$
\begin{aligned}
\overrightarrow{\mathcal{R}}^{\prime}(t) & =\left(\begin{array}{c}
\mathcal{R}_{x}^{\prime}(t) \\
\mathcal{R}_{y}^{\prime}(t)
\end{array}\right) \\
& =\left(\begin{array}{l}
\frac{\partial F_{1}(x, y)}{\partial x} \mathcal{R}_{x}(t)+\frac{\partial F_{1}(x, y)}{\partial y} \mathcal{R}_{y}(t) \\
\frac{\partial F_{2}(x, y)}{\partial x} \mathcal{R}_{x}(t)+\frac{\partial F_{2}(x, y)}{\partial y} \mathcal{R}_{y}(t)
\end{array}\right) .
\end{aligned}
$$

Evaluating the partial derivatives in Eqs. (1) and (2) yields differential equations explicitly in terms of the variables and parameters of the $\mathrm{FN}$ system:

$$
\begin{aligned}
\overrightarrow{\mathcal{R}}^{\prime}(t) & =\left(\begin{array}{c}
\mathcal{R}_{x}^{\prime}(t) \\
\mathcal{R}_{y}^{\prime}(t)
\end{array}\right) \\
& =\left(\begin{array}{c}
c\left(\left(1-x(t)^{2}\right) \mathcal{R}_{x}(t)-\mathcal{R}_{y}(t)\right) \\
\frac{1}{c}\left(\mathcal{R}_{x}(t)-b \mathcal{R}_{y}(t)\right)
\end{array}\right) .
\end{aligned}
$$

These differential equations are linear but contain, in the case of the first term of the $\mathcal{R}_{x}^{\prime}(t)$ equation, a

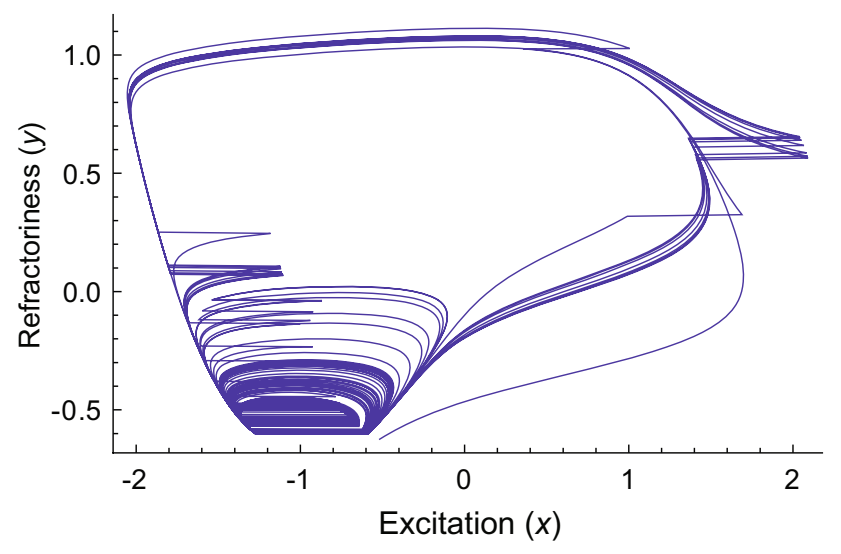

FIG. 2. Response of the FN model to high-rate stimulation. Parametric or "phase-plane" plot of the excitation $(x)$ and refractory (y) variables of the FN model. As a guide to the eye, horizontal line segments are drawn to connect the discontinuous segments of the solution. These discontinuities are due to the stimulus pulses. The stimulus rate is $5 \mathrm{kHz}$ and the stimulus level is 1.13278 times threshold $\left(A_{\theta}=0.602349\right)$. 
time-dependent coefficient. This coefficient is coupled to the evolution of the excitation variable $x(t)$, which in turn is governed by the nonlinear differential Eqs. (1) and (2). Because of this timedependent coefficient, these equations have no readily obtainable closed-form solutions (Hirsch and Smale 1974) and must be solved numerically, as in the case of the main $\mathrm{FN}$ equations. In our simulations, the combined system consisting of the main FN Eqs. (1) and (2), as well as the variational equations, summarized by the vector Eq. (5), was solved using algorithms described in the Computational Methods.

\section{The Lyapunov exponent}

The Lyapunov exponent characterizes the rate of growth of the magnitude of the perturbation vector, whose evolution is governed by the variational equations derived above (Eckmann and Ruelle 1985). Mathematically, the exponent is

$$
\lambda=\lim _{t \rightarrow \infty} \frac{1}{t} \ln \frac{|\overrightarrow{\mathcal{R}}(t)|}{|\overrightarrow{\mathcal{R}}(0)|}
$$

where $|\overrightarrow{\mathcal{R}}|=\sqrt{\mathcal{R}_{x}^{2}+\mathcal{R}_{y}^{2}}$. We estimate the Lyapunov exponent by solving the main and variational equations over a long but finite time interval $\left[t_{\min }, t_{\max }\right]$ and computing:

$$
\lambda \simeq \frac{1}{t_{\max }-t_{\min }} \ln \frac{\left|\overrightarrow{\mathcal{R}}\left(t_{\max }\right)\right|}{\left|\overline{\mathcal{R}}\left(t_{\min }\right)\right|}
$$

The values of $t_{\min }$ and $t_{\max }$ are chosen large enough to yield a robust estimate of the exponent while avoiding transients associated with stimulus onset.

\section{Simulating the effect of physiological noise}

To approximate the effects of the voltage noise generated by the random opening and closing of sodium channels (White et al. 2000), we added a stochastic noise term $\xi(t)$ to the differential equation for the excitation variable $x(t)$. For simplicity, we ignored any direct stochastic influences on the refractory variable $y(t)$. The noise term modifies the evolution of the perturbation through the dependence of the variational equation on $x(t)$. We note that changes in the short-term instability during the action potential modulate the effect of the constant-intensity noise $\xi(t)$, as shown in Supplemental Figure 8.

\section{Quantifying neural synchronization}

In this study, the mutual synchronization between pairs of spike trains was quantified by the spike-train cross-correlation histogram (Johnson and Kiang 1976; Dayan and Abbott 2001). As in Litvak et al. (2003a), these histograms were computed by forming a sequence of binary values $\left\{\beta_{n}\right\}$ for each of the spike trains, where $\beta_{n}=0$ if no spike occurs during the $n^{\text {th }}$ stimulus period and where $\beta_{n}=1$ otherwise. Superscripts $a$ and $b$ are used to distinguish the two binary sequences $\left\{\beta_{n}^{a}\right\}$ and $\left\{\beta_{n}^{b}\right\}$ associated with the two spike trains. The resulting cross-correlation histogram $H_{k}^{a, b}$ was then defined in terms of these binary sequences as

$$
N_{p} H_{k}^{a, b}=\sum_{n=0}^{N_{p}-|k|-1} \beta_{n}^{a} \beta_{n+k}^{b}-\frac{1}{N_{p}}\left(\sum_{n=0}^{N_{p}} \beta_{n}^{a}\right)\left(\sum_{n=0}^{N_{p}} \beta_{n}^{b}\right),
$$

where $N_{p}$ is the number of stimulus pulses. We note that $H_{k}^{a, b}$ quantifies the correspondence between the spike trains, but does not contain information concerning the timing of a spike within the stimulus period.

\section{Scaling the discharge rate}

When stimulated intensely at high rates, auditory nerve fibers initially fire rapidly $(>1,000$ spikes/s), but then adapt over tens of milliseconds to a lower rate of $\sim 400$ spikes/s (Moxon 1967; 1971). Like most auditory nerve models (e.g., Bruce et al. 1999 and Rubinstein et al. 1999), the standard FN model does not include a slow "adaptation" variable (FitzHugh 1969), and therefore exhibits considerably higher maximum sustained discharge rates than found in auditory nerve fibers (Fig. 8A).

To compare the rate dependence of the firing irregularity in the FN model with auditory nerve data, we normalize-out this difference in maximum discharge rate. Specifically, when plotting the empirically measured dependence of the firing irregularity on the discharge rate (Figs. 4 and 6), we express the discharge rate in dimensionless terms as $R / R_{u}$, where the "upper rate" $R_{u}$ is the rate at which the quantitative measure of irregularity, the Fano factor, drops below 0.05 on average in auditory nerve fibers $\left(R_{u}^{\mathrm{ANF}}=393\right.$ spikes $\left./ \mathrm{s}\right)$. The corresponding $\mathrm{FN}$ value is about three times larger $R_{u}^{\mathrm{FN}}=1,174$ spikes/s. To account for the distribution of maximum discharge rates observed in auditory nerve fibers (Zhang et al. 2007 ), this value $R_{u}^{\mathrm{FN}}$ was taken to be the mean value $\bar{R}_{u}^{\mathrm{FN}}$ of a population of otherwise identical model 
fibers. This distribution's normalized width was assumed to equal the normalized standard deviation of the maximum discharge rates, $\sigma_{\max } / \bar{R}_{\max }=0.343$, recorded in auditory nerve fibers (see the 100-ms condition of Fig. 4 of Zhang et al. 2007). The form of the distribution was assumed to be Gaussian for rates within \pm 2.5 standard deviations of the mean and to be zero outside of this range.

\section{Computational methods}

The deterministic FN Eqs. (1) and (2) along with the associated variational equations, summarized by the vector Eq. (5), were solved using the NDSolve algorithm of Mathematica 5 and 6 (Wolfram 2003). This algorithm dynamically adjusts the step size to produce solutions that meet a preset precision goal. Unless otherwise noted, this precision goal was set to eight decimal digits.

Adaptive-step-size methods have uncertain convergence properties when applied to stochastic differential equations (Longtin 2003). For this reason, the fixed-step-size stochastic Euler method was used to solve the FN equations with the additional stochastic noise term, along with the associated variational Eq. (5). This method was implemented in compiled Mathematica code with a standard machine precision of 16 decimal digits. The step size for most of these simulations was chosen to be $\Delta t=0.014$, which is approximately $1 \%$ of the spike upstroke time. We found that using smaller step

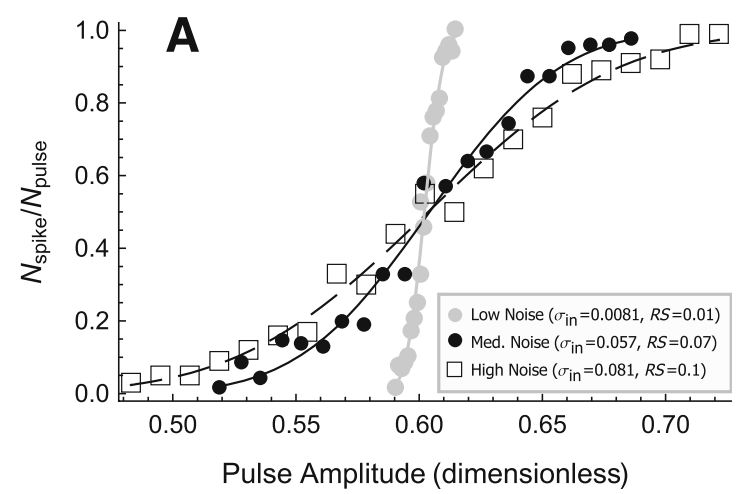

FIG. 3. Noise broadens the rate-level function to low-rate stimulation. A Firing rate versus pulse amplitude for noise intensities producing typical (black points, black solid line), low (gray points, gray line), and high (open boxes, dashed line) values of the relative spread (see figure legend for details). The stimulus is a low-rate $\left(T=10 t_{d}\right)$ pulse train consisting of 100 pulses. The pulse amplitude $A$ is expressed in the units of Eq. (1) and the firing rate $(R)$ is expressed as the fraction of stimulus pulses eliciting a spike $\left(N_{\text {spike }} / N_{\text {pulse }}\right)$. Sigmoidal curves represent two-parameter leastsquare fits of the cumulative Gaussian function expressed in terms of the error function $R\left(A ; A_{50 \%}, \sigma_{\text {out }}\right)=\frac{1}{2}\left[1+\operatorname{Erf}\left(\frac{A-A_{50 \%}}{\sqrt{2 \sigma_{\text {out }}}}\right)\right]$. The sizes did not significantly affect the results (e.g., Supplemental Figs. 12 and 13).

\section{RESULTS}

Ongoing noise accounts for irregularity at low stimulation rates

Here, we adjust the level of the noise in the FN model so that the range of stimulus amplitudes producing irregular firing at low rates matches those observed in auditory nerve measurements. Subsequently, we will demonstrate that this level of ongoing noise is inconsistent with the firing irregularity observed at high stimulation rates.

At low stimulation rates, and in the absence of noise, a uniform pulse train evokes either no firing at all or firing to every pulse, depending on whether the stimulus level is below or above threshold. If noise is present, however, a range of near-threshold pulse amplitudes evokes probabilistic, irregular firing (Verveen and Derksen 1968; White et al. 2000). The range of stimulus levels evoking irregular firing at low rates is quantified by the relative spread $(R S)$, defined as the ratio of half of the width of the rate-level function to the pulse amplitude that produces a $50 \%$ firing probability (Verveen and Derksen 1968; White et al. 2000). The rate-level functions of auditory nerve fibers when stimulated by low-rate pulse trains have $R S$ values of $0.01-0.1$ (Dynes 1996), with a median value of approximately 0.07 (Miller et al. 2001).

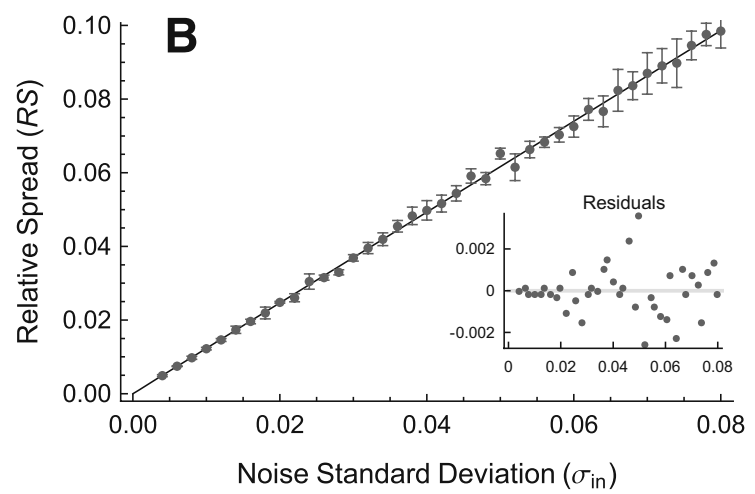

inflection point of the sigmoid is determined by the parameter $A_{50 \%}$ and the sigmoid's width by $\sigma_{\text {out }}$. B The relative spread $\left(R S \equiv \sigma_{\text {out }} / A_{50 \%}\right)$ as a function of the input noise level $\left(\sigma_{\text {in }}\right)$. Points represent the mean of five trials and the error bars indicate the standard deviation. A linear least-squares fit constrained to go through the origin is represented by the straight line. The bestfitting slope is 1.2332. The residuals about this fit are shown in the inset panel. The estimate of the uncertainty in the $R S$ is

$\Delta_{R S} \equiv \sqrt{\frac{1}{N-2} \sum_{i=1}^{N}\left(R S_{i}-f_{\mathrm{fit}}\left(\sigma_{\mathrm{in}_{i}}\right)\right)^{2}}=1.366 \times 10^{-6}$. 
Examples of rate-level functions produced by the FN model in response to low-rate pulse trains $\left(T=10 t_{d}\right)$ are shown in Figure 3A for three different noise levels. The lines represent cumulative Gaussian fits to the data achieved using a least-squares procedure. This fit has two parameters, the width or "spread" $\sigma_{\text {out }}$ and the $50 \%$ firing level $A_{50 \%}$, the ratio of which determines the relative spread $R S \equiv \sigma_{\text {out }} / A_{50 \%}$. Figure 3B presents the value of the relative spread as a function of the input noise level $\sigma_{\mathrm{in}}$. A linear dependence of the $R S$ on the input noise level is predicted by the analytic theory of Lecar and Nossal (1971a) and by biophysically detailed computational simulations which incorporate probabilistic descriptions of individual sodium channels (Clay and DeFelice 1983; Rubinstein 1995). Figure 3 demonstrates that the FN model with ongoing noise reproduces the sigmoidal rate-level functions that are observed in auditory nerve fibers when stimulated at low rates.

Ongoing noise fails to reproduce non-Poisson-like firing at high stimulation rates

The firing irregularity produced by high-rate $(\sim 5 \mathrm{kHz})$ electrical stimulation has been quantified by Litvak et al. (2001) and by Miller et al. (2008). The measure of irregularity used in these physiological studies was the normalized spike count variance or Fano factor $(F F)$. The Fano factor is calculated by counting the number of spikes $N_{k}$ within each of $k$ bins of duration $T_{c}$ and dividing the variance of these counts by the mean count:

$$
F F \equiv \operatorname{Var}\left[N_{k}\right] / \operatorname{Mean}\left[N_{k}\right] .
$$

The Fano factors measured by Litvak et al. (boxes) and Miller et al. (diamonds) are shown in Figure 4 as a function of normalized discharge rate $R / R_{u}$ (see Methods for a definition of $R_{u}$ and the rationale for scaling the discharge rate). These physiological measurements show that the Fano factor tends to fall outside the range of values predicted by the dead-time modified Poisson process. In contrast, the predictions of the FN model with noise (blue points; $R S=0.07$ ) agree closely with the dead-time modified Poisson process (gray). Loess trend lines show that the FN model with noise tends to underestimate the Fano factor at low rates and overestimate it at high rates. Although the trend of the FN model only slightly overestimates the data trend at intermediate discharge rates near $R / R_{u} \simeq 0.5$, the $\mathrm{FN}$ predictions substantially underestimate the scatter of the data about the trend line. Scaling the FN data to account for the range of measured maximum discharge rates (see Methods) does not alter the tendency for the FN trend to be too low at low rates and too high at high rates, although it does improve the prediction of the downward (but not appreciably the upward) disper-

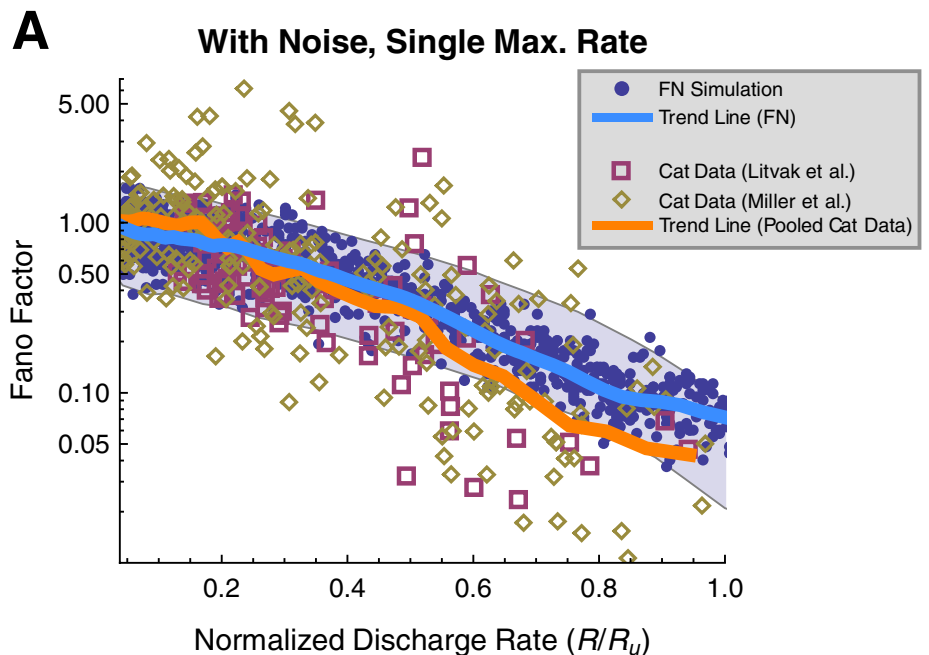

\section{B With Noise, Distribution of Max. Rates}

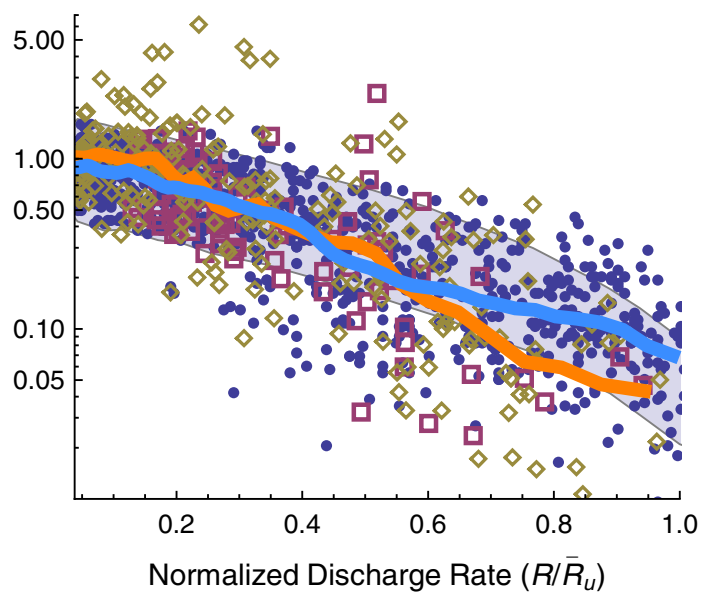

FIG. 4. The FN model with noise fails to predict the irregular response of auditory nerve fibers. A The Fano factor versus the discharge rate for electrically stimulated auditory nerve fibers (Litvak et al. 2001 data, open boxes; Miller et al. 2008 data, open diamonds) and the FN model with ongoing noise (points). In $\mathbf{B}$, predictions of the FN model are scaled to account for the distribution of maximum discharge rates measured in electrically stimulated auditory nerve fibers (Zhang et al. 2007). Curves represent loess trend line fits (Cleveland 1993) to the auditory nerve data (combined across experiments) and to the FN data. The auditory data consists of a total of 300 data points collected from 114 fibers (Litvak et al. 2001; Miller et al. 2008). Approximately 950 data points are shown for the FN simulations. Points within the gray region fall between the $1 \%$ and $99 \%$ quantiles of a dead-time modified Poisson process. The modified Poisson dead times are $2 \mathrm{~ms}$ for the auditory nerve data, as in Litvak et al. (2001) and Miller et al. (2008), and $0.67 \mathrm{~ms}$ for the FN model. Values of $R_{u}\left(\bar{R}_{u}\right.$ in B) are 393 spikes/s and 1,174 spikes/s for the auditory nerve data and the FN model, respectively. 


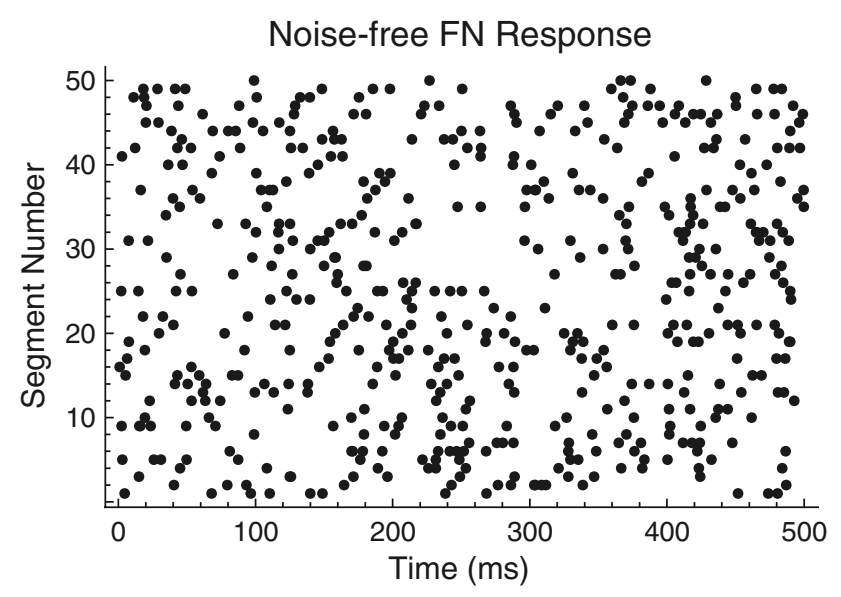

FIG. 5. An irregular discharge pattern produced by the noise-free FN model. A dot raster plot showing the spike times predicted by the noise-free $\mathrm{FN}$ model. As with the auditory nerve response shown in Figure 1, the raster plot represents the spiking response in 50 contiguous $500 \mathrm{~ms}$ time windows. The stimulus is a $5-\mathrm{kHz}$ pulse train.

sion at intermediate rates. Evidently, when the noise level of the FN model is adjusted to produce a typical value of the relative spread to low-rate stimulation (Fig. 3), it is incapable of predicting the non-Poissonlike firing irregularity exhibited by auditory nerve fibers at high stimulation rates.
Eliminating noise improves aspects of the $\mathrm{FN}$ model's irregularity prediction

A surprising feature of the response of the FN model is that it can be irregular even in the absence of ongoing noise (Ermentrout 1984; Feingold et al. 1988; Rajasekar and Lakshmanan 1988, 1994; Sato and Doi 1992; Rabinovitch et al. 1994; Doi and Sato 1995; Kaplan et al. 1996; Rabinovitch and Rogachevskii 1999). Here, we show that completely eliminating ongoing noise improves prominent aspects of the fit to the auditory nerve data.

An example of the irregular response of the FN model when noise is removed is shown in Figure 5 (cf. the auditory nerve spike-time raster in Fig. 1). In Figure 6 , the firing irregularity produced by the noisefree $\mathrm{FN}$ model is quantified as a function of the discharge rate and is compared to the auditory nerve data. In contrast to the with-noise $\mathrm{FN}$ response (Fig. 4A), the noise-free FN response (Fig. 6A) spans a more realistic range of Fano factors, although it does not account for the largest Fano factors recorded by Miller et al. (2008). As the trend line indicates, eliminating noise raises the Fano factor at low discharge rates and decreases it at high discharge rates. When scaled to account for the range of maximum discharge rates (Fig. 6B), the noise-free FN model produces a good fit to the data trend for a range of low rates $\left(0.1<R / R_{u}<0.2\right)$ and at rates
A

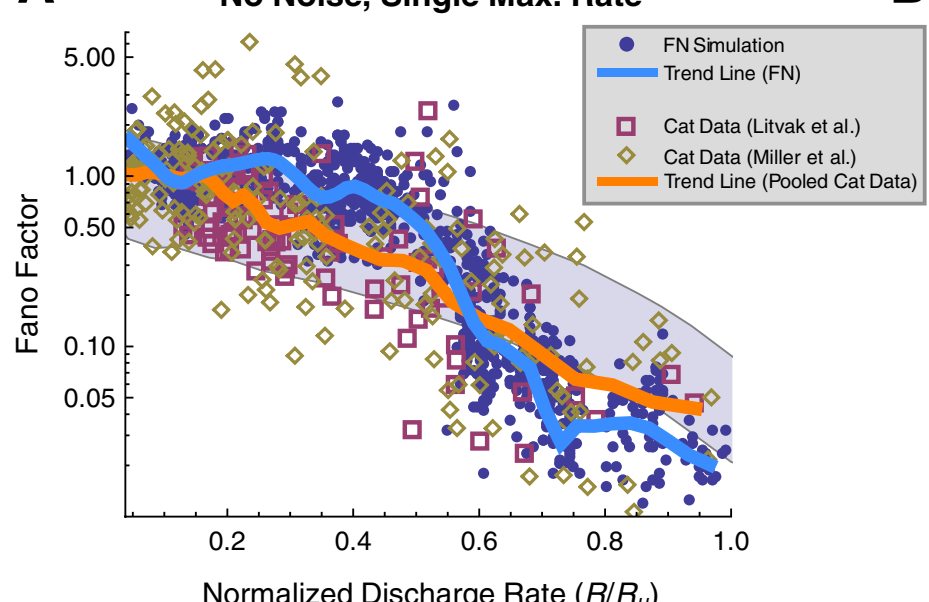

B

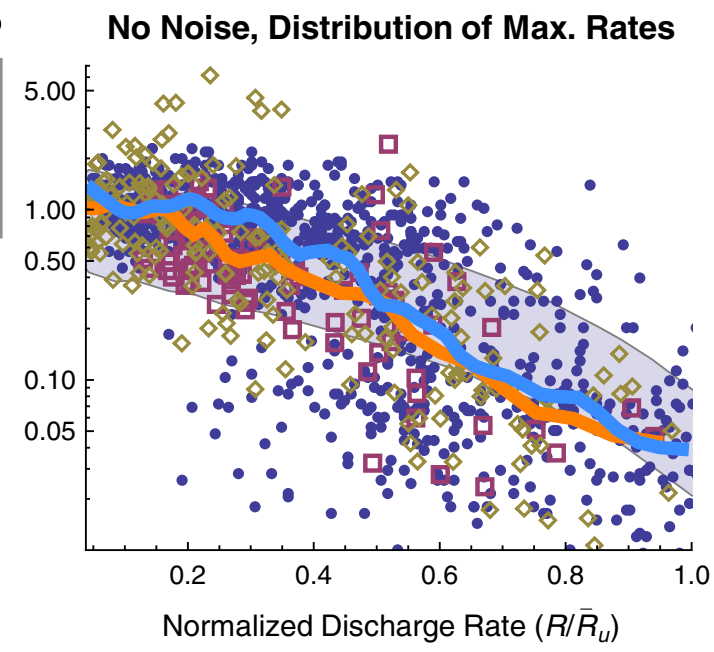

FIG. 6. Removing ongoing noise improves aspects of the FN model's irregularity prediction. A The Fano factor versus the discharge rate for electrically stimulated auditory nerve fibers (Litvak et al. 2001 data, open boxes; Miller et al. 2008 data, open diamonds) and the FN model without ongoing noise (points). In panel $\mathbf{B}$, predictions of the FN model are scaled to account for the distribution of maximum discharge rates measured in electrically stimulated auditory nerve fibers (Zhang et al. 2007). Curves represent loess trend line fits (Cleveland 1993) to the auditory nerve data (combined across experiments) and to the $\mathrm{FN}$ data, respectively. The auditory data consists of a total of 300 data points collected from 114 fibers (Litvak et al. 2001; Miller et al. 2008). Approximately 950 data points are shown for the FN simulations. Points within the gray region fall between the 1\% and $99 \%$ quantiles of a deadtime modified Poisson process. The modified Poisson dead times are $2 \mathrm{~ms}$ for the auditory nerve data, as in Litvak et al. (2001) and Miller et al. (2008), and $0.67 \mathrm{~ms}$ for the $\mathrm{FN}$ model. Values of $R_{u}\left(\bar{R}_{u}\right.$ in $\left.\mathbf{B}\right)$ are 393 spikes/s and 1,174 spikes/s for the auditory nerve data and the FN model, respectively. 
above about 0.47 . For these higher rates, the noise-free FN model also predicts nearly the full range of scatter about the trend. For the lowest rates $\left(R / R_{u}<0.1\right)$ and for intermediate rates $\left(0.2<R / R_{u}<0.47\right)$ the noisefree FN model predicts more irregularity on average than is apparent either in the data or in the with-noise FN response (Fig. 4). This general phenomenon in which removing noise increases the irregularity of a system has been observed previously in nonlinear maps (Mayer-Kress and Haken 1981) and has been referred to as "noise-induced order" (Matsumoto and Tsuda 1983; Matsumoto 1984; Doi 1989). We note that unlike in "coherence resonance" (Pikovsky and Kurths 1997), noise reduces the coherence of the response (Supplemental Figs. 9 and 10). ("Coherence resonance" refers to the regularization and correlation produced as an intense noise pushes the discharge rate toward the inverse of the refractory time.)

Dynamical instability produces the irregularity observed in the absence of ongoing noise

We now investigate the mechanism by which irregularity is produced in the FN model even in the absence of ongoing noise. A dynamically unstable system has the property that the response $|\overrightarrow{\mathcal{R}}(t)|$ to a small perturbation grows over time (Eckmann and Ruelle 1985). Dynamical instability produces irregularity by amplifying the irregularity present in the digits of low significance in the initial conditions and in the system parameters. In other words, dynamical instability converts "microscopic" irregularity that is present initially into "macroscopic" irregularity manifest over time.

The time evolution of the response to a small perturbation $|\overrightarrow{\mathcal{R}}(t)|$ is governed by the variational equations, which are derived from the main FN Eqs. (1) and (2) as described in Methods. The time evolution of the voltage-like variable $x(t)$ and the perturbed response $|\overrightarrow{\mathcal{R}}(t)|$ are shown in Figure 7 . The short-term increase in $|\overrightarrow{\mathcal{R}}(t)|$ apparent primarily during the spike upstroke, spike downstroke, and during subthreshold responses (Fig. 7A and B) leads to longterm growth of the perturbed response $\overrightarrow{\mathcal{R}}(t)$ (Fig. 7D) and to a noticeably irregular firing pattern (Fig. 7C). The approximately linear growth of the logarithm of $|\overrightarrow{\mathcal{R}}(t)| /|\overrightarrow{\mathcal{R}}(0)|$ evident in Figure 7D demonstrates that $|\overrightarrow{\mathcal{R}}(t)|$ increases exponentially on average. The rate of this exponential growth (or decay) defines the Lyapunov exponent $\lambda$. In this example, the response grows at a rate of $\lambda \simeq 1.76 \mathrm{~ms}^{-1}$, as indicated by the straight line in Figure 7D. The power-law convergence of estimates of the Lyapunov exponent is illustrated in the Supplemental Methods, along with demonstrations of the robustness of the estimates to computational error. Further details about the definition of the Lyapunov exponent are given in the Supplemental Note. In the Supplemental Computations, we also show that short-term expansion in
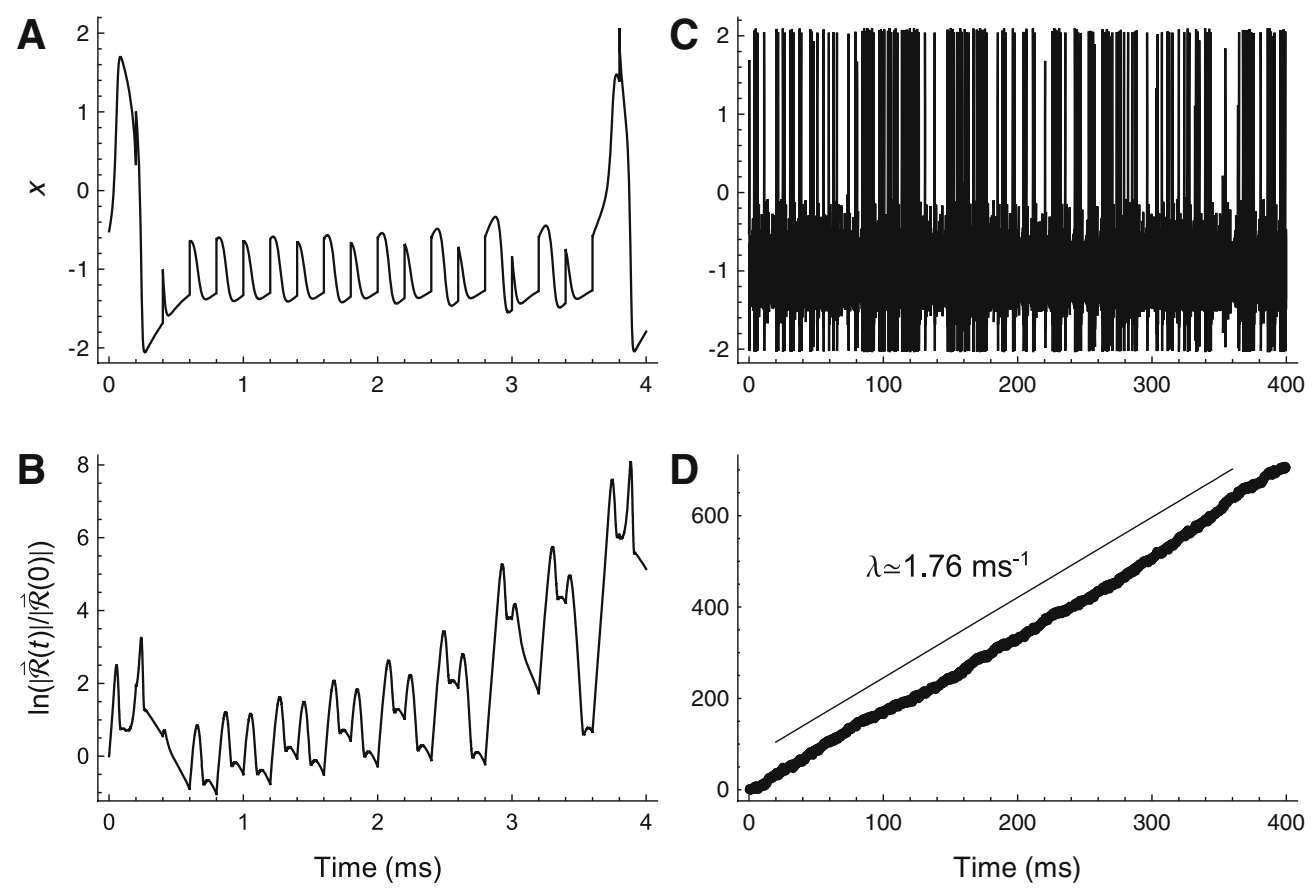

FIG. 7. Long-term growth in the response to a small perturbation produces firing irregularity at late times. The response of the voltage-like variable $x$ of the FN model over short (A) and long (C) time scales and the corresponding log of the magnitude of the

perturbed response $|\overrightarrow{\mathcal{R}}(t)|$ (B, D). The size of the perturbed response is expressed in terms of the initial perturbation size $|\overrightarrow{\mathcal{R}}(0)|$. The stimulus rate is $5 \mathrm{kHz}$ and the stimulus level is 1.13278 times threshold, as in Figure 2. 

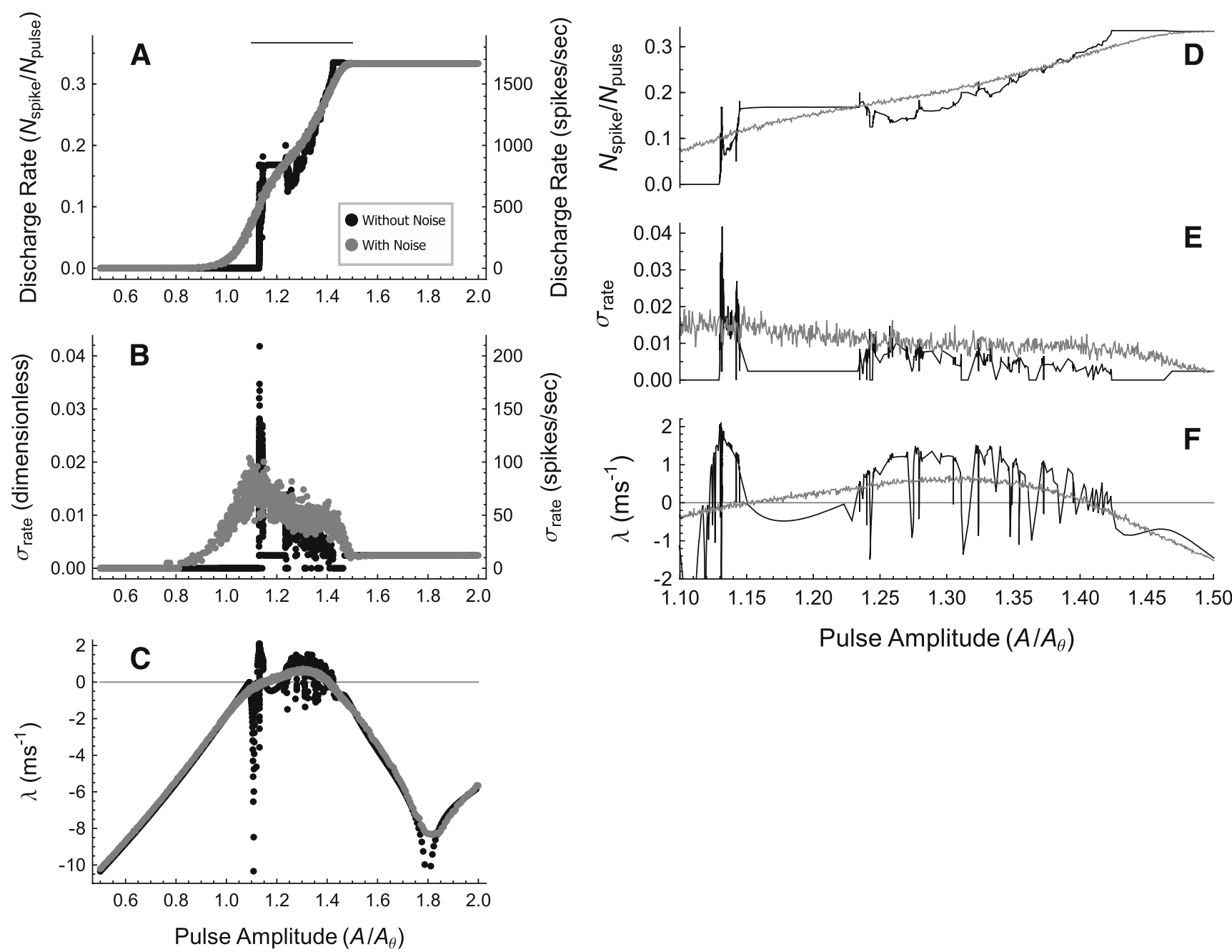

FIG. 8. The Lyapunov exponent determines the sensitivity to level increments and the effect of ongoing noise. Plots of the discharge rate $N_{\text {spike }} / N_{\text {pulse }}(\mathbf{A})$, the standard deviation of the discharge rate $\sigma_{R}(A)(\mathbf{B})$, and the Lyapunov exponent $\lambda(A)(\mathbf{C})$ as a function of the stimulus level $A / A_{\theta}$ for the $\mathrm{FN}$ model driven at $5 \mathrm{kHz}$. In these plots, $N_{\text {spike }}$ is the number of spikes, $N_{\text {pulse }}$ is the number of stimulus pulses, and $A_{\theta}$ is the neural threshold defined as the minimum pulse amplitude required to produce a spike at a low stimulation rate. Black points/curves represent the noise-free results and gray points/ curves represent the results with ongoing noise. The intensity of the

$|\overrightarrow{\mathcal{R}}(t)|$ amplifies the noise-induced fluctuations in $x$ (Supplemental Fig. 8) and that similar short-term expansion occurs in more complex biophysical models (Supplemental Fig. 5).

We measure "dynamical instability" using the value of the Lyapunov exponent. Thus, if $\lambda_{1}>\lambda_{2}$, we say that the system described by $\lambda_{1}$ is more "unstable" or has greater "dynamical instability" than the system described by $\lambda_{2}$, even if $\lambda_{1}<0$.

Finally, we note that the preceding discussion of the relationship between the voltage $x(t)$ and the perturbed response $|\overrightarrow{\mathcal{R}}(t)|$ is consistent with and complementary to the earlier analysis of the high-rate

noise is adjusted to produce a physiologically typical relative spread of $R S=0.07$. Panels $\mathbf{D}-\mathbf{F}$ zoom in on the range of pulse amplitudes in the peak region of $\lambda$ between $A / A_{\theta}=1.10$ and $A / A_{\theta}=1.5$, where $\lambda>0$ typically. This range of levels is indicated by the black bar above $\mathbf{A}$. The estimates of $\lambda$ and $R$ represent the mean of the estimates from 24 contiguous segments of the stimulus, each consisting of 200 pulses. A 100-pulse initial segment was discarded to minimize the influence of dynamic transients on the subsequent estimates. Calculations of $\sigma_{\mathrm{R}}$ were determined by estimating the firing rate associated with each of the 24 contiguous segments.

response of the FN model based on one-dimensional maps (Rabinovitch et al. 1994; Kaplan et al. 1996).

Dynamical instability determines the dependence of the firing rate and firing irregularity on stimulus level

Although it is well known that positive dynamical instability $\lambda>0$ implies response irregularity (e.g., Sato and Doi 1992), the relationship between the dynamical instability of a neural-spike generator and the resulting firing irregularity has not been quantified to our knowledge. The relationship between the degree of 
dynamical instability and the sensitivity to small finite increments in the stimulus intensity has also not been investigated in detail (cf. Fig. 8 of Ichinose et al. 1998 for which $\lambda<0$ almost always). To elucidate these relationships, we compute the discharge rate $R$ (Fig. $8 \mathrm{~A}$ and $\mathrm{D}$ ), the standard deviation of the discharge rate $\sigma_{R}$ (Fig. 8B and $\mathrm{E})$, and the Lyapunov exponent estimate $\lambda(A)$ (Fig. 8C and F) as a function of level $A / A_{\theta}$ of the stimulating pulse train, where $A / A_{\theta}$ is the pulse amplitude relative to the neural threshold defined as the minimum pulse amplitude required to produce a spike at a low stimulation rate. The interpulse time was fixed to $\hat{T}=0.2 \mathrm{~ms}$, a value near that used in recent studies of the electrically stimulated auditory nerve (Rubinstein et al. 1999; Litvak et al. 2001, 2003a, b, c; Miller et al. 2001; Zhang et al. 2007; Miller et al. 2008).

For the noise-free case (black), positive segments of $\lambda(A)$ correspond to segments of $R(A)$ for which the rate is changing with level, whereas negative segments of $\lambda(A)$ correspond to plateaus in $R(A)$ for which level increments produce no change in the firing rate, as with, for example, the $\operatorname{dip}$ in $\lambda(A)$ at $A / A_{\theta} \simeq 1.18$ and the corresponding plateau in $R(A)$. This relationship is most readily apparent in the right panels (Fig. 8D and $F$ ) which zoom in on a range of stimulus levels for which the Lyapunov exponent is typically positive. These simulations show that dynamical instability can allow for relatively smooth modulation of the firing rate by the stimulus level even in the absence of ongoing noise (we note also that the rate-level function $R(A)$ of the noise-free $\mathrm{FN}$ model; Fig. 8A and $\mathrm{D}$, black; is similar to that measured in squid axons, Fig. 12 of Takahashi et al. 1990).

Likewise, segments in which the standard deviation of the firing rate $\sigma_{R}$ (Fig. 8B, E) is large coincide with positive segments of $\lambda(A)$. When $\lambda(A)$ is negative, $\sigma_{R}(A)$ is constant, with a value at or near zero. The constant, non-zero values are due to beating of the periodic firing pattern with the analysis bin duration (note that if the spike count is zero for every analysis bin, we define $\sigma_{R}(A)$ to be zero). These simulations show that the Lyapunov exponent largely determines the standard deviation of the firing rate in the absence of ongoing noise.

Dynamical instability determines the effect of ongoing physiological noise

The effect of noise (gray points and curves in Fig. 8) is to smooth the noise-free results, lowering sharp peaks, raising sharp valleys, and eliminating plateaus. For example, in the presence of noise, the peaks and dips in $\lambda(A)$ between $A / A_{\theta}=1.1$ and $A / A_{\theta}=1.5$ are smoothed-out, leaving only a single broad peak that lacks fine structure (Fig. 8F). Likewise, steep regions of the rate-level function, corresponding to high increment sensitivity, and the plateaus of the ratelevel function, corresponding to low increment sensitivity, are smoothed-out by noise.

The tendency of noise to smooth or minimize fluctuations by lowering sharp peaks and raising sharp valleys is also evident when the stochastic prediction of $\sigma_{R}(A)$ (gray) is compared to the noise-free prediction of $\sigma_{R}(A)$ (black). In particular, noise raises $\sigma_{R}(A)$ significantly above zero near $A / A_{\theta} \simeq 1$ and $A / A_{\theta} \simeq 1.18$, while in the absence of noise $\sigma_{R}$ is essentially zero for these conditions. Likewise, the noise markedly reduces the amplitude of the prominent peak in $\sigma_{R}(A)$ that is apparent in the noise-free simulations near $A / A_{\theta} \simeq 1.13$.

Despite the smoothing effect of noise, dynamical instability largely determines the increment sensitivity and the firing irregularity, as evidenced by the close correspondence between the broad peak in the Lyapunov exponent (gray; Fig. 8C and F) and the range of stimulus levels over which the firing changes with level (gray; Fig. 8A and D) and is irregular (gray; Fig. 8B and E). Unlike the noise-free case, however, firing can be irregular even when the spike generator is stable $(\lambda<0)$, so long as it is not too stable, as it is for stimulus levels above $A / A_{\theta} \simeq 1.5$. We note that in addition to the Lyapunov exponent, the noise-tostimulus-level ratio, which decreases with stimulus level in Figure 8, also likely contributes to the firing irregularity, which probably explains why the noise has a somewhat smaller effect at high stimulus levels for the same value of $\lambda$.

\section{Dynamical instability accounts for the dependence of the firing irregularity on the discharge rate}

Since dynamical instability largely determines the dependence of the firing irregularity on the stimulus level (Fig. 8), one might expect that it also plays a large role in determining the dependence of the Fano factor on the discharge rate. We test this idea in Figure 9 by plotting the Fano factor $(\mathrm{A}, \mathrm{C})$ and Lyapunov exponent (B, D) as a function of the discharge rate.

The noise-free plots (Fig. 9A and B) show that at low rates $\left(R / R_{u}<0.47\right)$, when the Lyapunov exponent is larger than the firing rate, the Fano factor is within or above predictions of the dead-time modified Poisson process, although one point in this range is associated with a nearly zero Lyapunov exponent and, correspondingly, a nearly zero Fano factor. At higher rates $\left(R / R_{u}>0.47\right)$, when the Lyapunov exponent is either smaller than the firing rate or is negative, the Fano factor typically falls within or below the predictions of the modified Poisson process. In addition, a prominent local minimum in the Fano factor 
FN Model, No Noise
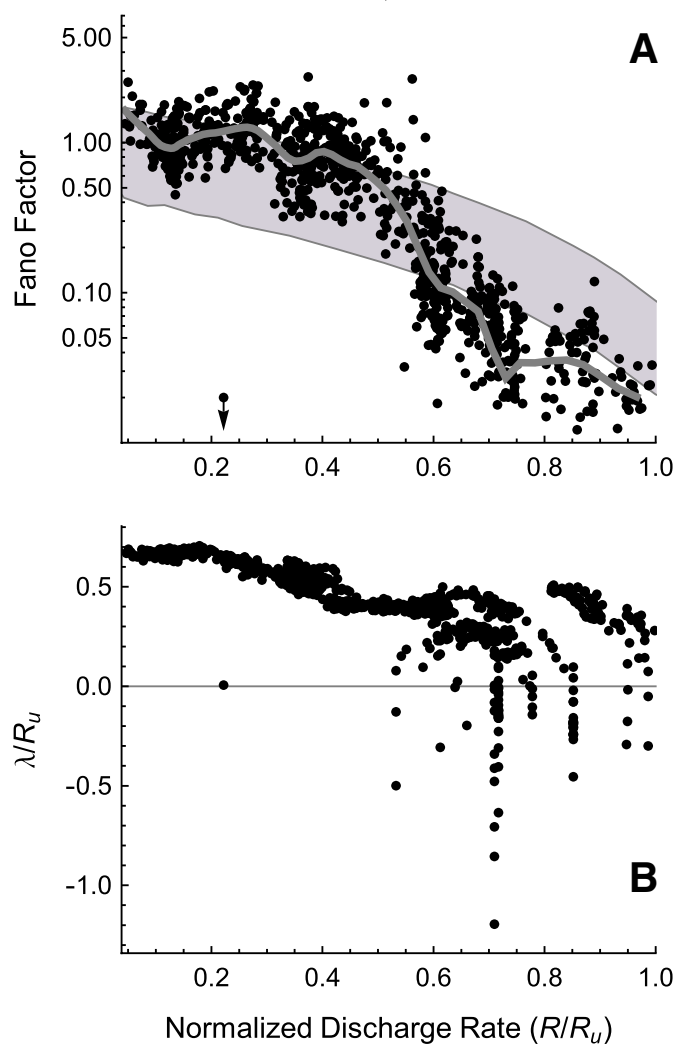

FIG. 9. Dynamical instability produces non-Poisson-like firing irregularity. The estimate of the Fano factor $(\mathbf{A}, \mathbf{C})$ and Lyapunov exponent (B, D) versus the discharge rate for the $\mathrm{FN}$ model without and with ongoing noise $(R S=0.07)$. Gray curves indicate loess trend

occurring near $R / R_{u}=0.72$ and a local maximum in the Fano factor occurring near $R / R_{u}=0.86$ correspond approximately to minima and maxima, respectively, in the Lyapunov exponent.

In the presence of noise, a not-too-negative Lyapunov exponent $-0.8<\lambda / R_{u}<0$ produces Poisson-like firing at low rates $\left(R / R_{u}<0.6\right)$. At the higher discharge rates, a positive Lyapunov exponent greatly slows the decreasing trend of the Fano factor, in opposition to the predictions of the dead-time modified Poisson process.

These simulations show that both in the absence and in the presence of noise, the discharge-rate dependence of $\lambda$-the $\lambda(R)$ profile-shapes the discharge-rate dependence of the Fano factor.

Dynamical instability determines the dependence of firing irregularity on the stimulation rate

Figures 8 and 9 show the relationship between the dynamical instability and the firing irregularity for a fixed stimulation rate. Here, we investigate the Fano factor (Fig. 10A) and the degree of dynamical instability (Fig. 10B) as a function of the interpulse
FN Model, With Noise
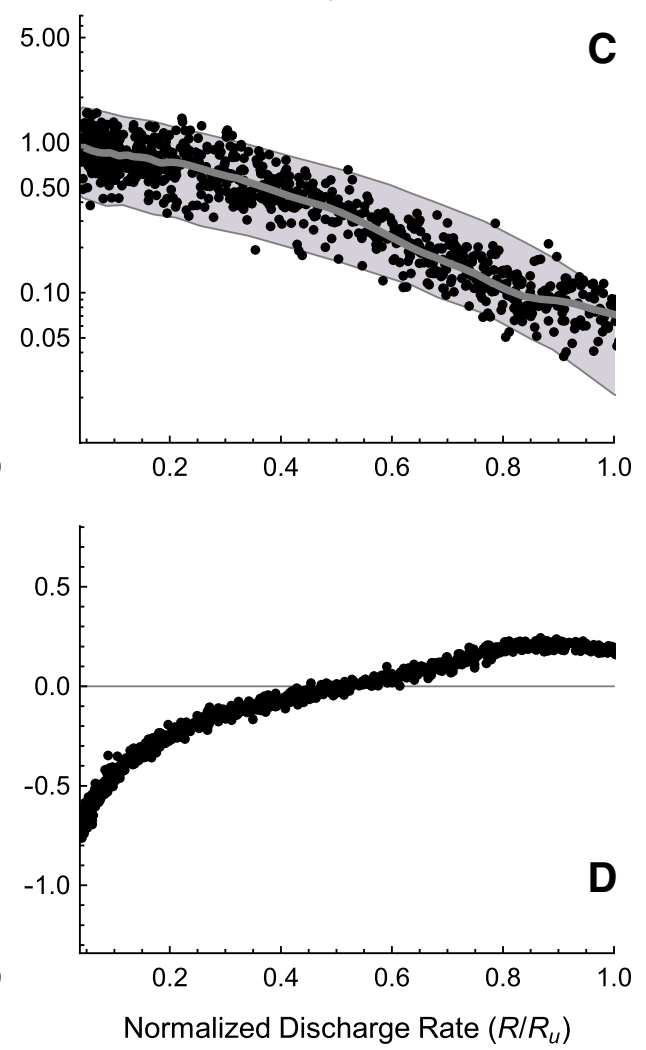

line fits $(\mathbf{A}, \mathbf{C})$. As in Figures 4 and 6, points within the gray region in C and $\mathbf{D}$ fall between the $1 \%$ and $99 \%$ quantiles of the best-fitting dead-time modified Poisson process. Approximately 950 data points are shown for the deterministic and stochastic FN simulations.

time for a fixed stimulus level. The interpulse time $T$ is varied from 0.1 to 5 times the nominal refractory time $t_{d}$, defined to be the spike downstroke time, and the stimulus level is fixed at about 1.13 times the threshold.

In the noise-free case, peaks in the Lyapunov exponent (Fig. 10B, black) produce non-zero Fano factors. Note that no point is plotted in Figure 10B when the Fano factor is below about 0.001 or when the Fano factor is undefined because the firing rate is zero, as it typically is for $0.1 \leq T / t_{d} \leq 1$. A similar dependence of the Lyapunov exponent on the interpulse time has been reported previously in one-dimensional map approximations to the FN equations (Ichinose et al. 1998; Coombes and Osbaldestin 2000).

Ongoing noise (gray) smoothes the dependence of the Lyapunov exponent on the interpulse time and causes firing irregularity to occur even when the Lyapunov exponent is large and negative. The value of the Fano factor is nevertheless modulated by the dynamical instability, so that the major peaks in the Lyapunov exponent correspond to the major peaks in the Fano factor. Thus, stimulus-rate-dependent changes in the Lyapunov exponent produce stimu- 

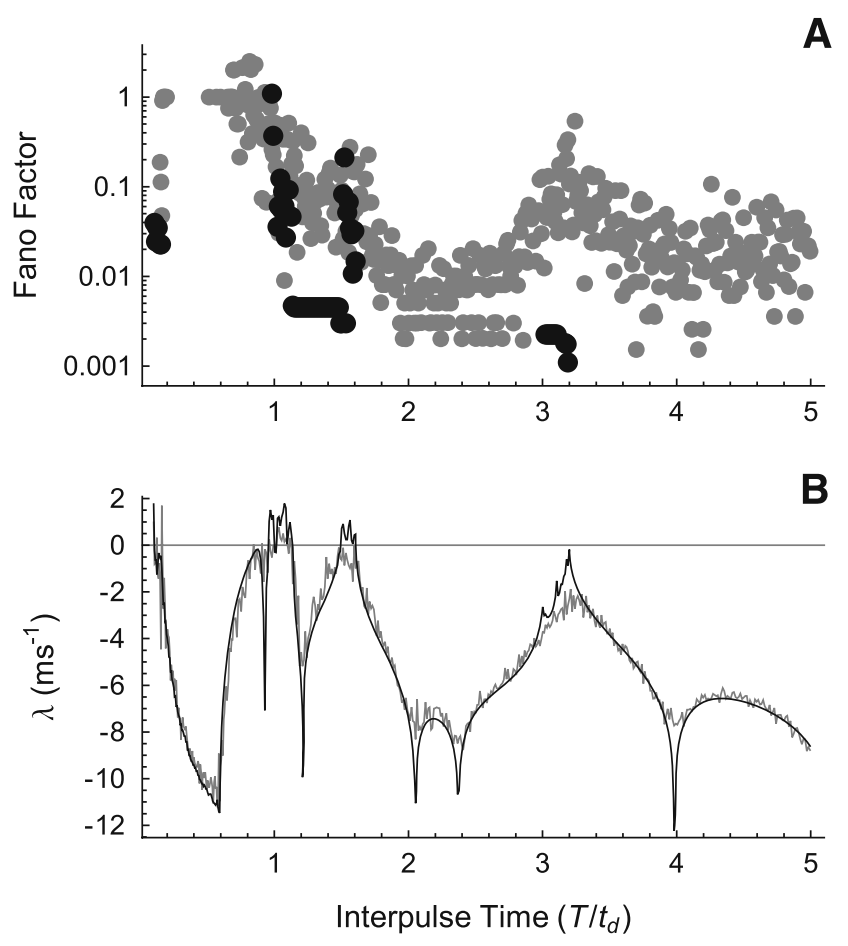

FIG. 10. The Lyapunov exponent determines the dependence of the firing irregularity on stimulation rate. The Fano factor $(\mathbf{A})$ and Lyapunov exponent $\lambda(T)(\mathbf{B})$ versus the interpulse time $T$ for the $\mathrm{FN}$ model without noise (black) and with noise (gray; $R S=0.07$ ). The interpulse time is expressed in units of the spike downstroke time $t_{d}$. Note that no point is plotted if the Fano factor is less than about 0.001 or is undefined because the firing rate is zero. The stimulus level is 1.13278 times threshold as in Figures 2 and 7.

lus-rate-dependent changes in the firing irregularity, even in the presence of ongoing noise (the desynchronization produced at high stimulation rates by a positive Lyapunov exponent is treated in the Discussion section).

\section{DISCUSSION}

Changes in Lyapunov exponent can produce rate-dependent changes in noise sensitivity

All accounts of auditory nerve fiber responses to highrate pulse trains of which we are aware attribute the observed firing irregularity to the effects of channel noise (Rubinstein et al. 1999; Litvak et al. 2001, 2003a; Miller et al. 2008). This standard explanation, however, is incomplete since it does not specify why noise has different effects at different stimulation rates (Moxon 1967; van den Honert and Stypulkowski 1987; Javel 1990; Javel and Viemeister 2000).

Our analysis of the simple FN model of neural excitation and refractoriness identifies a mechanism that can produce stimulus-rate-dependent changes in the firing irregularity. Specifically, we demonstrate that the dynamical instability, quantified by the Lyapunov exponent, exhibits prominent fluctuations as the interpulse time $T$ is reduced below about ten times the refractory time (Fig. 10 and Supplemental Figs. 1 and $4 \mathrm{a}$ ) and that these fluctuations modulate the firing irregularity. Similar rate-dependent fluctuations in the Lyapunov exponent also occur in more complicated biophysical models (Supplemental Figs. 2, 3, and 4b,c) and in one-dimensional map approximations to the FN model (Ichinose et al. 1998; Coombes and Osbaldestin 2000). The Lyapunov exponent determines not only the sensitivity to infinitesimal pulse-like perturbations, but also the response to ongoing noise (Figs. 8 and 10). At high stimulus rates, but not at low, the model's deterministic nonlinear dynamics create an instability $(\lambda>0)$ that produces high sensitivity both to initial conditions (Fig. 7D) and to small, finite perturbations (Fig. 8A and D).

Dynamical instability accounts for features of the non-Poisson-like response of auditory nerve fibers stimulated at high rates

Somewhat surprisingly given the simplicity of the FN model, we find that dynamical instability $(\lambda>0)$ can account for the main features of the firing irregularity observed experimentally at high stimulation rates in auditory nerve fibers (Fig. 6). This agreement occurs even in the complete absence of ongoing physiological noise. Indeed, physiological noise of only moderate intensity substantially reduces the firing irregularity and the prominence of the non-Poisson-like features of the response (Fig. 9C). This regularization reflects the noise-induced averaging of the Lyapunov exponents associated with nearby trajectories in the phase-plane (Fig. 2), which in turn smoothes the level dependence of the Lyapunov estimate, lowering its peaks and raising its valleys (Fig. 8C and F). To our knowledge, this regularizing role of physiological noise in auditory nerve responses has not previously been recognized. We note that the regularization reported in the context of "coherence resonance" (Pikovsky and Kurths 1997) occurs when a very intense noise pushes the discharge rate toward the inverse of the refractory time, whereas the regularization we report occurs at relatively low noise levels and discharge rates (Fig. 9A and $\mathrm{C}$ ).

A dynamical-stability-based explanation of the failure of the FN model to simultaneously account for irregularity at high and low stimulus rates

Our results show that the FN model is incapable of simultaneously reproducing the firing irregularity observed at low and high stimulation rates in auditory 
nerve fibers. Specifically, additive noise of sufficient intensity to produce a realistic relative spread at low stimulation rates (Fig. 3) prevents the FN model from reproducing the non-Poisson-like behavior at high stimulation rates (Fig. 4). This discrepancy suggests that the dynamical stability of the FN model at low stimulation rates is unrealistically high (i.e., the Lyapunov exponent is too negative). Were the Lyapunov exponent less negative at these low stimulation rates, a lower noise intensity would be sufficient to produce a realistic relative spread, and the nonPoisson-like character of the response at high stimulation rates might then be preserved.

Furthermore, the FN model is apparently incapable of accounting for the non-Poisson-like behavior observed at stimulation rates as low as $250 \mathrm{~Hz}$ (Miller et al. 2008), since our simulations suggest that this non-Poisson behavior requires dynamical instability (Fig. 9), $\lambda>0$, and the FN model evidently does not produce dynamical instability at these low simulation rates (Fig. 10 and Supplemental Fig. 1).

We speculate that slow dynamical processes (e.g., Hindmarsh and Rose 1982, 1984; Rinzel and Ermentrout 1998), absent in the standard FN model, may account for these discrepancies by decreasing the dynamical stability (i.e., making $\lambda$ less negative) at low stimulation rates and by producing instability, $\lambda>0$, at intermediate stimulation rates. These slow dynamical mechanisms might also account for the lower maximum firing rate of auditory nerve fibers compared with the FN model, account for slow changes in the discharge patterns that occur after stimulus onset (Litvak et al. 2001, 2003a; Zhang et al. 2007; Miller et al. 2008), and for highly irregular responses (Fano factor near 5) reported by Miller et al. (2008). Javel and Viemeister (2000) have previously suggested that slow processes were necessary to produce the highly irregular responses that they observed. Slow voltageand calcium-dependent conductances identified in auditory nerve fibers presumably contribute to these slow dynamical mechanisms (Chen 1997; Mo and Davis 1997; Adamson et al. 2002).

Implications for physiology underlying dynamic range and modulation sensitivity in auditory prostheses

Although the Lyapunov exponent strictly quantifies only the system's sensitivity to infinitesimal perturbations about a reference response, the results of Figure 8 show that the Lyapunov exponent also correlates strongly with sensitivity to small but finite increments in the stimulus level. Specifically, when the exponent is large and negative the response is insensitive to small increases in the stimulus level. But when the exponent is positive or near zero, small changes in the stimulus level produce changes in the firing rate. The Lyapunov exponent and its variation with stimulus level are therefore likely to play major roles in determining the dynamic range of the neural response.

We conjecture that the Lyapunov exponent $\lambda$ determines the sensitivity not only to small pulsatile and step-like perturbations, but also to modulations of arbitrary shape, provided that the modulations are relatively small in amplitude and slow compared to the Lyapunov time $1 / \lambda$. Consistent with this conjecture, FN model simulations (O'Gorman et al. 2007) show that dynamical instability can account for the extremely high sensitivity (i.e., modulation thresholds of $<0.5 \%$ ) of auditory fibers to slow sinusoidal amplitude modulations (Litvak et al. 2003b). Since sensitivity to sinusoidal amplitude modulation correlates strongly with speech perception in both cochlear implantees (Fu 2002) and patients with brain stem implants (Colletti and Shannon 2005), the mechanisms that determine the Lyapunov exponent, therefore, may be critical for speech perception using these devices.

We speculate, for example, that dynamical instabilities of the sort described here might determine critical parameters of temporal processing that are thought to underlie speech perception in cochlear implant patients (Fu and Shannon 2000). These parameters include the minimum carrier rate $(\sim 200 \mathrm{~Hz})$ needed to readily convey amplitude modulations and the maximum modulation rate perceivable as a distinct pitch $(\sim 500 \mathrm{~Hz})$. In this view, the minimum carrier rate would correspond to the lowest stimulation rate that produces dynamical instability in auditory nerve fibers and the maximum modulation rate would be on the order of the value of $\lambda$.

\section{Dynamical instability produces desynchronization with respect to the stimulus}

In addition to producing nearly the full range of observed firing irregularity (Fig. 6A), dynamical instability also accounts for the observation, in both auditory nerve models (Rubinstein et al. 1999) and experiments (Litvak et al. 2001), that neural responses to high-rate pulse trains remain synchronized to the stimulus for the first few spikes, but desynchronize after about $5 \mathrm{~ms}$. We show in the Supplemental Computations (Supplemental Fig. 9) that in the FN model the time to desynchronize corresponds to roughly ten times the Lyapunov time $1 / \lambda$. In our simulations, memoryless noise of the kind invoked to account for desynchronization (Rubinstein et al. 1999; Litvak et al. 2003a) substantially shortens the initial period of synchronized firing (Supplemental Fig. 10; we note that that these simulations also 
show that the noise is not inducing "coherence resonance", Pikovsky and Kurths 1997).

\section{Dynamical instability produces cross-fiber desynchronization}

The auditory nerve consists of tens of thousands of individual auditory nerve fibers, but the extent to which each fiber functions as an independent channel for auditory information depends on the degree of statistical decorrelation or desynchronization across the neural population (Johnson and Kiang 1976). Litvak et al. (2003a) measured the responses of pairs of fibers simultaneously driven by a common $5 \mathrm{kHz}$ pulse train and showed that these responses were consistent with mutual desynchronization apart from the fine-timing synchrony to each stimulus pulse. Similarly desynchronized responses have been observed in healthy fibers, both in response to silence and to acoustic tones (Johnson and Kiang 1976; Kiang 1990).

In these experiments, the degree of cross-fiber synchronization was quantified by the spike-train cross-correlation histogram. An example of a crosscorrelation histogram constructed from the data set of
Litvak et al. (2003a) is shown in Figure 11A. In these histograms, a value of zero indicates desynchronization, and the statistical significance of fluctuations about zero is determined by comparison to the corresponding renewal process. A single realization of this renewal process is generated by randomly shuffling the intervals in the two spike trains and computing the cross-correlation histogram from these shuffled spike trains. If the random fluctuations about zero are statistically indistinguishable from those produced by this renewal process, then the spike trains are considered to be mutually desynchronized. The $1 \%$ and $99 \%$ quantiles for the corresponding renewal processes are indicated by the gray points in Figure 11A. The distribution of the amplitudes taken on by the cross-correlation histogram is plotted in Figure 11D, along with the ranges of the amplitudes computed from 100 realizations of the renewal process (gray points).

Like the auditory nerve data, the fluctuations in the cross-correlation histogram predicted by the noisefree FN model (Fig. 11B and E) are statistically similar to the corresponding renewal process. In the absence of ongoing noise, this statistical independence is
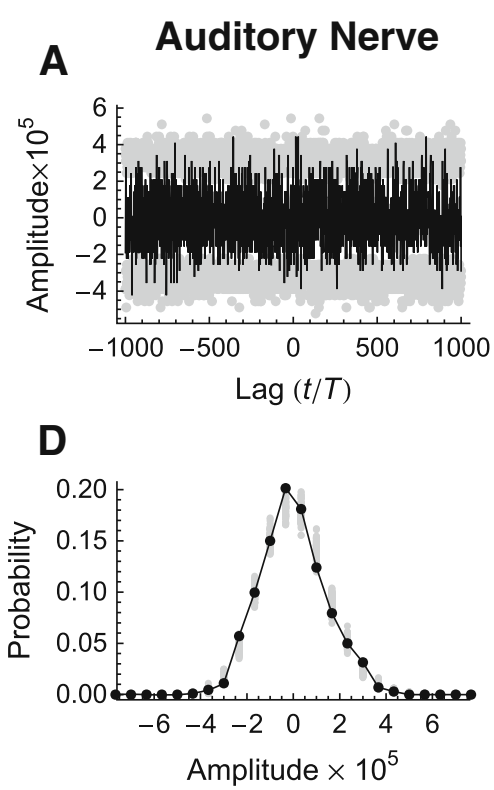

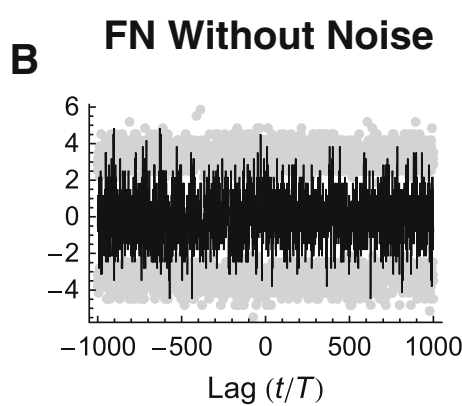

E

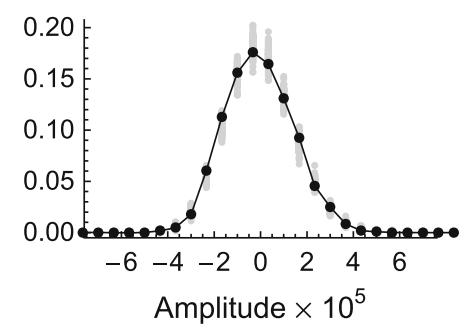

FN With Noise

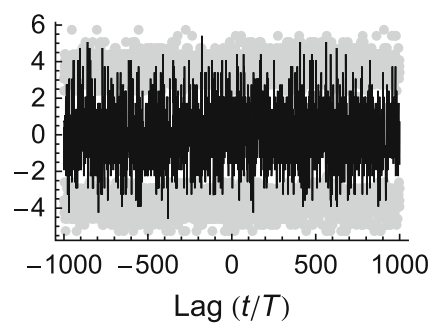

$\mathbf{F}$

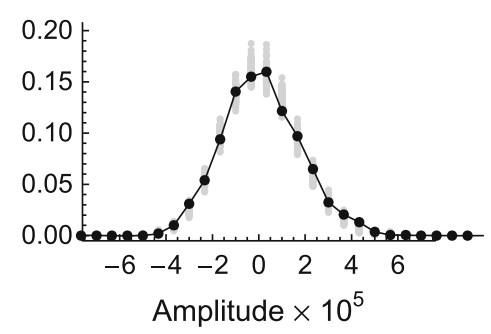

FIG. 11. Realistic cross-fiber desynchronization produced by a positive Lyapunov exponent in the noise-free $\mathrm{FN}$ model. Spike-train cross-correlation histograms $(\mathbf{A}-\mathbf{C})$ and corresponding crosscorrelation amplitude density histograms (D-F) constructed from spike trains recorded simultaneously from a pair of electrically stimulated auditory nerve fibers (A) and from a pair of responses produced by the $\mathrm{FN}$ model without ongoing noise (B) and with ongoing noise $(\mathbf{C} ; R S=0.07)$. The stimulus is a $5-\mathrm{kHz}$ pulse train. The physiological data are from the set collected by Litvak et al. (2003a). The gray points in A-C indicate the $1 \%$ and $99 \%$ quantiles of the corresponding renewal process, while the gray points in $\mathbf{D}-\mathbf{E}$ indicate the amplitudes taken on in 100 realizations of the corresponding renewal process. The firing rates for the two auditory nerve fibers are $18.6 \pm 1.6$ and $83.55 \pm 9.36$. For the $\mathrm{FN}$ simulations, the level of the pulse train was adjusted to produce firing rates that approximately matched those of the experimental pair. Specifically, the rates are $20.8 \pm 1.7 \mathrm{spikes} / \mathrm{s}\left(A / A_{\theta}=1.12992\right)$ and $77.92 \pm 2.35 \mathrm{spikes} / \mathrm{s}\left(A / A_{\theta}=\right.$ $1.12998)$ for the noise-free $\mathrm{FN}$ simulations and $22.95 \pm 1.77$ spikes $/ \mathrm{s}$ $\left(A / A_{\theta}=0.953\right)$ and $86.3 \pm 2.6$ spikes $/ \mathrm{s}\left(A / A_{\theta}=1.013\right)$ for the simulations with noise. The Lyapunov exponents are $\lambda \simeq 2.05 \mathrm{~ms}^{-1}$ and $\lambda \simeq 1.195 \mathrm{~ms}^{-1}$ for the noise-free simulations and $\lambda \simeq-2.72 \mathrm{~ms}^{-1}$ and $\lambda \simeq-1.58 \mathrm{~ms}^{-1}$ for the simulations with noise. 
produced entirely by dynamical instability. The results from the FN model with noise (Fig. 11C and F) show that renewal-like desynchronization also occurs when the Lyapunov exponent is negative if the noise level is sufficiently high. We note, however, that were the spike generator even more stable (i.e., the Lyapunov exponent more negative), as it is at higher stimulus levels (Fig. 8C), the response would be both highly regular (Fig. 8B) and synchronized across fibers, despite the ongoing noise.

The desynchronization observed with high-rate electrical stimulation has been attributed to noise (Rubinstein et al. 1999) and/or to fiber-to-fiber differences in refractoriness, sensitivity, or other properties (Litvak et al. 2001). As with firing irregularity, these explanations are incomplete since they do not specify why noise and cross-fiber differences have different effects at different stimulation rates (Moxon 1967; van den Honert and Stypulkowski 1987; Javel 1990; Javel and Viemeister 2000). Our simulations demonstrate that stimulus-rate-dependent increases in the Lyapunov exponent can cause cross-fiber desynchronization at high stimulation rates even in the complete absence of fiber-to-fiber differences in refractoriness, sensitivity, and other properties. In addition, our simulations show that dynamical instability $(\lambda>0)$ can produce cross-fiber desynchronization even in the complete absence of ongoing noise.

Our results from the FN model also suggest a novel resolution to a paradox or "oddment" (Kiang 1990) concerning the firing behavior of healthy auditory fibers, namely, that even though cochlear excitation patterns are spatially broad, presumably driving many fibers simultaneously, firing patterns from fibers that innervate the same region of the cochlea are nevertheless mutually independent (Johnson and Kiang 1976). One possible resolution suggested by Johnson and Kiang (1976) is that the broad peripheral excitation "might only modulate the activity of a very localized but noisy excitatory process, and it is this excitatory process that triggers the spike discharges" (p. 730). Our work shows that the paradox can be resolved without invoking noise, if one assumes that the excitatory process is sufficiently rapid to produce dynamical instability $(\lambda>0)$ at the spike generation site. The extraordinary sensitivity of fibers to faint sounds (Kiang et al. 1965) and the presence of measurable correlations between sequential intervals in the spontaneous discharge pattern (Lowen and Teich 1992) are further consistent with the excitatory process being low-noise, and perhaps controlled by dynamical instability, since dynamical instability produces high sensitivity (Fig. 8) and produces correlated firing on the time scale of $1 / \lambda$ (Supplemental Fig. 9). Determining which hypothesis is correct may require measuring the Lyapunov exponent of the auditory nerve fiber's spike generator.

\section{Dynamical instability of the neural-spike generator}

Like healthy auditory nerve fibers, many neurons in the nervous system fire in an irregular and desynchronized fashion (Dayan and Abbott 2001). Irregular and desynchronized neural firing is usually attributed to irregular and desynchronized synaptic input (Dayan and Abbott 2001) or to physiological noise (White et al. 2000). Our work, however, shows that firing irregularity and desynchronization depend not only on the characteristics of the effective input to the neuron, but also on the dynamical instability of the spike generator. Although irregularity in synaptic transmission has been well studied (Siegel 1992; Faure and Korn 1997; Heil et al. 2007), the dynamical response of the target neuron has received comparatively little attention. Our results indicate that this aspect of synaptic transmission warrants further study. For example, recent recordings at the afferent terminal of primary auditory fibers show that the inner hair cell synapse is capable of rapid vesicle release (Glowatzki and Fuchs 2002). Based on our results, we speculate that rapid spontaneous transmitter release maintains the receiving fiber in a state of dynamical instability $(\lambda>0)$, thereby enhancing the sensitivity and temporal responsiveness of the auditory nerve.

\section{ACKNOWLEDGMENTS}

We are grateful to Leo Litvak, Zachary Smith, and Charles Miller for making their data available to us. We would also like to acknowledge Ian Bruce, Steve Colburn, Bertrand Delgutte, Don Eddington, John Guinan, Danny Kaplan, Nelson Kiang, Nancy Kopell, Jennifer Melcher, Rob Morse, Bill Peake, Jay Rubinstein, Erik Sherwood, Nigel Stocks, and John Wyatt for stimulating discussions.

Grants This work was supported by a Speech and Hearing Science Training Grant from the NIDCD (T32-DC00038), the Kirschstein Award (NIH F31DC006188), an MIT-Poitras Fellowship, and the MIT-Zakhartchenko Fellowship. Additional support was provided by the Burroughs-Wellcome Fund, a grant from the NIH to J.A. White (R01-NS34425), and the Hearing Research Center at Boston University.

\section{REFERENCES}

Adamson CL, Reid MA, Mo ZL, Bowne-English J, Davis RL. Firing features and potassium channel content of murine spiral ganglion neurons vary with cochlear location. J. Comp. Neurol. 447:331-350, 2002. 
Aihara K, Matsumoto G, Ikegaya Y. Periodic and non-periodic responses of a periodically forced Hodgkin-Huxley oscillator. J. Theor. Biol. 109:249-269, 1984.

BonHoEffer KF. Activation of passive iron as a model for the excitation of nerve. J. Gen. Physiol. 32:69-91, 1948.

Bruce IC, Irlicht LS, White MW, O’Leary SJ, Dynes S, Javel E, Clark GM. A stochastic model of the electrically stimulated auditory nerve: pulse-train response. IEEE Trans. Biomed. Eng. 46:630-637, 1999.

Chen C. Hyperpolarization-activated current (I-h) in primary auditory neurons. Hear. Res. 110:179-190, 1997.

Clay JR, DeFelice LJ. Relationship between membrane excitability and single channel open-close kinetics. Biophys. J. 42:151-157, 1983.

CLAY JR. Excitability of the squid giant axon revisited. J. Neurophysiol. 80:903-913, 1998.

Cleveland WS. Visualizing Data. Summit, New Jersey, Hobart, 1993.

Colletti V, Shannon RV. Open set speech perception with auditory brainstem implant. Laryngoscope 115:1974-1978, 2005.

Coombes S, Osbaldestin AH. Period-adding bifurcations and chaos in a periodically stimulated excitable neural relaxation oscillator. Phys. Rev. E 62:4057-4066, 2000.

Dayan P, Aвbоtт LF. Theoretical neuroscience: computational and mathematical modeling of neural systems. Cambridge, Mass, MIT Press, p. 460, 2001.

Dor S. A chaotic map with a flat segment can produce a noiseinduced order. J. Stat. Phys. 55:941-964, 1989.

Dor SJ, SATO S. The global bifurcation structure of the BVP neuronal model driven by periodic pulse trains. Math. Biosci. 125:229250, 1995.

Dynes SBC. Discharge characteristics of auditory nerve fibers for pulsatile electrical stimuli. PhD Thesis, MIT, Cambridge, MA, 1996.

Eckmann J-P, Ruelle D. Ergodic theory of chaos and strange attractors. Rev. Mod. Phys. 57:617-656, 1985.

Ermentrout GB. Period doublings and possible chaos in neural models. SIAM J. Appl. Math. 44:80-95, 1984.

Faure P, Korn H. A nonrandom dynamic component in the synaptic noise of a central neuron. Proc. Natl. Acad. Sci. USA 94:65066511, 1997.

Feingold M, Gonzales DL, Piro O, Viturro H. Phase locking, period doubling, and chaotic phenomena in externally driven excitable systems. Phys. Rev. A 37:4060-4063, 1988.

FitzHugh F. Mathematical models of excitation and propagation in nerve. In: Schwan HP (ed) Biological Engineering. New York, McGraw-Hill, 1969.

FitzHugh R. Impulses and physiological states in theoretical models of nerve membrane. Biophys. J. 1:445-465, 1961.

Fu QJ, Shannon RV. Effect of stimulation rate on phoneme recognition by Nucleus-22 cochlear implant listeners. J. Acoust. Soc. Am. 107:589-597, 2000.

Fu QJ. Temporal processing and speech recognition in cochlear implant users. Neuroreport 13:1635-1639, 2002.

Glowatzki E, Fuchs PA. Transmitter release at the hair cell ribbon synapse. Nat. Neurosci. 5:147-154, 2002.

Guckenheimer J, Oliva RA. Chaos in the Hodgkin-Huxley model. SIAM J. Appl. Dyn. Syst. 1:105-114, 2002.

Heil P, Neubauer H, Irvine DRF, Brown M. Spontaneous activity of auditory-nerve fiber stochastic processes at ribbon synapses. J. Neurosci. 27:8457-8474, 2007.

HindmaRsh JL, Rose RM. A model of the nerve impulse using two first-order differential equations. Nature 296:162-164, 1982.

HindmaRsh JL, Rose RM. A model of neuronal bursting using 3 coupled 1st order differential-equations. Proc. R. Soc. Lond. B Biol. Sci. 221:87-102, 1984.

Hirsch MW, Smale S. Differential Equations, Dynamical Systems, and Linear Algebra. New York, Academic, 1974.

Hochmair-Desoyer IJ, Hochmair ES, Motz H, Rattay F. A model for the electrostimulation of the nervus acusticus. Neuroscience 13:553-562, 1984.
Hodgkin AL, Huxley AF. A quantitative description of membrane current and its application to conduction and excitation in nerve. J. Physiol. 117:500-544, 1952.

Hong RS, Rubinstein JT. High-rate conditioning pulse trains in cochlear implants: Dynamic range measures with sinusoidal stimuli. J. Acoust. Soc. Am. 114:3327-3342, 2003.

ICHinose N, Ainara K, Judd K. Extending the concept of isochrons from oscillatory to excitable systems for modeling an excitable neuron. Int. J. Bifurcat. Chaos 8:2375-2385, 1998.

JAVEL E. Acoustic and electrical encoding of temporal information. In: Miller JM, Spelman FA (eds) Cochlear Implants: Models of the Electrically Stimulated Ear. New York, Springer, pp. 247-292, 1990.

Javel E, Viemeister NF. Stochastic properties of cat auditory nerve responses to electric and acoustic stimuli and application to intensity discrimination. J. Acoust. Soc. Am 107:908-921, 2000.

Johnson DH, KIANG NY. Analysis of discharges recorded simultaneously from pairs of auditory nerve fibers. Biophys. J. 16:719734,1976

Kaplan DT, Clay JR, Manning T, Glass L, Guevara Mr, Shrier A. Subthreshold dynamics in periodically stimulated squid giant axons. Phys. Rev. Lett. 76:4074-4077, 1996.

Kepler TB, Авbott LF, Marder E. Reduction of conductance-based neuron models. Biol. Cybern. 66:381-387, 1992.

Kiang NY, Watanabe T, Thomas EC, Clark LF. Discharge Patterns of Single Fibers in the Cat's Auditory Nerve. Cambridge, MIT Press, 1965.

KIANG NY. Curious oddments of auditory-nerve studies. Hear. Res. 49:1-16, 1990.

Kosmidis EK, Pakdaman K. Stochastic chaos in a neuronal model. Int. J. Bifurcat. Chaos 16:395-410, 2006.

Lecar H, Nossal R. Theory of threshold fluctuations in nerves: I. Relationships between electrical noise and fluctuations in axon firing. Biophys. J. 11:1048-1067, 1971a.

Lecar H, Nossal R. Theory of threshold fluctuations in nerves: II. Analysis of various sources of membrane noise. Biophys. J. 11:1068-1084, 1971b.

LiN KK. Entrainment and chaos in a pulse-driven Hodgkin-Huxley oscillator. SIAM J. Appl. Dyn. Syst. 5:179-204, 2006.

Litvak LM, Delgutte B, Eddington DK. Auditory-nerve fiber responses to electric stimulation: Modulated and unmodulated pulse trains. J. Acoust. Soc. Am. 110:368-379, 2001.

Litvak LM, Smith ZM, Delgutte B, Eddington DK. Desynchronization of electrically evoked auditory-nerve activity by highfrequency pulse trains of long duration. J. Acoust. Soc. Am. 114:2066-2078, 2003a.

Litvak LM, Delgutte B, Eddington DK. Improved temporal coding of sinusoids in electric stimulation of the auditory nerve using desynchronizing pulse trains. J. Acoust. Soc. Am. 114:2079-2098, 2003b.

Litvak LM, Delgutte B, Eddington DK. Improved neural representation of vowels in electric stimulation using desynchronizing pulse trains. J. Acoust. Soc. Am. 114:2099-2111, 2003c.

LONGTin A. Effect of noise on nonlinear dynamics. In: Beuter A, Glass L, Mackey MC, and Titcombe M (eds) Nonlinear Dynamics in Physiology and Medicine. New York, 2003.

LoRenz EN. Deterministic nonperiodic flow. J. Atmos. Sci. 20:130141, 1963.

Lowen SB, Teich MC. Auditory-nerve action potentials form a nonrenewal point process over short as well as long time scales. J. Acoust. Soc. Am. 92:803-806, 1992.

Matsumoto G, Aihara K, Hanyu Y, Takahashi N, Yoshizawa S, Nagumo J. Chaos and phase locking in normal squid axons. Phys. Lett. A 123:162-166, 1987.

Matsumoto K, Tsuda I. Noise-induced order. J. Stat. Phys. 31:87-106, 1983.

Matsumoto K. Noise-induced order II. J. Stat. Phys. 34:111-127, 1984. 
Matsuoka AJ, Rubinstein JT, Abbas PJ, Miller CA. The effects of interpulse interval on stochastic properties of electrical stimulation: Models and measurements. IEEE Trans. Biomed. Eng. 48:416-424, 2001.

Mayer-Kress G, Haken H. The influence of noise on the logistic model. J. Stat. Phys. 26:149-171, 1981.

Miller CA, Abbas PJ, Robinson BK. Response properties of the refractory auditory nerve fiber. J. Assoc. Res. Otolaryngol. 2:216232, 2001.

Miller CA, Hu N, Zhang F, Robinson BK, Abbas PJ. Changes across time in the temporal responses of auditory-nerve fibers stimulated by electric pulse trains. J. Assoc. Res. Otolaryngol. 9:122137, 2008.

Mo ZL, DAVIS RL. Endogenous firing patterns of murine spiral ganglion neurons. J. Neurophysiol. 77:1294-1305, 1997.

Morse RP, Evans EF. Enhancement of vowel coding for cochlear implants by addition of noise. Nat. Med. 2:928-932, 1996.

Moss F, Chiou-Tan F, Kuinke R. Will there be noise in their ears. Nat. Med. 2:860-862, 1996.

Moxon EC. Electric stimulation of the cat's cochlea: A study of discharge rates in single auditory nerve fibers. Masters Thesis, MIT, Cambridge, MA, 1967.

Moxon EC. Neural and mechanical responses to electric stimulation of the cat's inner ear. Ph.D. Thesis, MIT, Cambridge, MA 1971.

Nagumo J, Авimoto S, Yoshizawa S. An active pulse transmission line stimulating nerve axon. P. IRE 50:2061-2070, 1963.

O'Gorman DE, Shera CA. Does the high-rate response of electrically stimulated neurons reflect nonclassical sodium gating? Assoc. Res. Otolaryngol. Abs. 24, 2001.

O'Gorman DE, Shera CA, White JW, Colburn HS. Realistically irregular and sensitive neural firing without physiological noise [abstract]. Proceedings of the 2007 Conference on Implantable Auditory Prostheses. Lake Tahoe, Nevada, 2007. p 139. Abstract B34.

Othmer HG, XIE M. Subharmonic resonance and chaos in forced excitable systems. J. Math. Biol. 39:139-171, 1999.

PIKovsky AS, KurThs J. Coherence resonance in a noise-driven excitable system. Phys. Rev. Lett. 78:775-778, 1997.

Rabinovitch A, Thieberger R, Friedman M. Forced Bonhoeffer-van der Pol oscillator in its excited mode. Phys. Rev. E 50:1572-1578, 1994.

Rabinovitch A, Rogachevski I. Threshold, excitability and isochrones in the Bonhoeffer-van der Pol system. Chaos 9:880-886, 1999.
Rajasekar S, Lakshmanan M. Period doubling route to chaos for a BVP oscillator with periodic external force. J. Theor. Biol. 133:473-477, 1988.

Rajasekar S, Lakshmanan M. Bifurcation, chaos and suppression of chaos in FitzHugh-Nagumo nerve conduction model equation. J. Theor. Biol. 166:275-288, 1994.

Rinzel J, Ermentrout GB. Analysis of neural excitability and oscillations. In: Koch C, Segev I (eds) Methods in Neuronal Modeling. Cambridge MA, MIT Press, pp. 251-291, 1998.

RUBinstein JT. Threshold fluctuations in an $\mathrm{N}$ sodium channel model of the node of Ranvier. Biophys. J. 68:779-785, 1995.

Rubinstein JT, Wilson BS, Finley CC, Abbas PJ. Pseudospontaneous activity: stochastic independence of auditory-nerve fibers with electrical stimulation. Hear. Res. 127:108-118, 1999.

Sakmann B, Neher E. Single-Channel Recording. New York, Plenum, 1983.

SATo S, Dor S. Response characteristics of the BVP neuron model to periodic pulse inputs. Math. Biosci. 112:243-259, 1992.

Siegel JH. Spontaneous synaptic potentials from afferent terminals in the guinea pig cochlea. Hear. Res. 59:85-92, 1992.

Sigworth FJ. The variance of sodium current fluctuations at the node of Ranvier. J. Physiol. (Lond) 307:97-129, 1980.

Stocks NG, Allingham D, Morse RP. The application of suprathreshold stochastic resonance to cochlear implant coding. Fluct. Noise Lett. 2:L169-L181, 2002.

Takahashi N, Hanyu Y, Musha T, Kubo R, Matsumoto G. Global bifurcation structure in periodically stimulated giant axons of squid. Physica D 43:318-334, 1990.

van den Honert C, STypulkowski PH. Temporal response patterns of single auditory nerve fibers elicited by periodic electrical stimuli. Hear. Res. 29:207-222, 1987.

Verveen AA, Derksen HE. Fluctuation phenomena in nerve membrane. P. IEEE 56:906-916, 1968.

White JA, Rubinstein JT, Kay AR. Channel noise in neurons. Trends Neurosci. 23:131-137, 2000.

Wolfram S. The Mathematica Book. Wolfram Media/Cambridge University Press, 2003.

Zhang F, Miller CA, Robinson BK, Abbas PJ, Hu N. Changes across time in spike rate and spike amplitude of auditory nerve fibers stimulated by electric pulse trains. J. Assoc. Res. Otolaryngol. 8:356-372, 2007. 